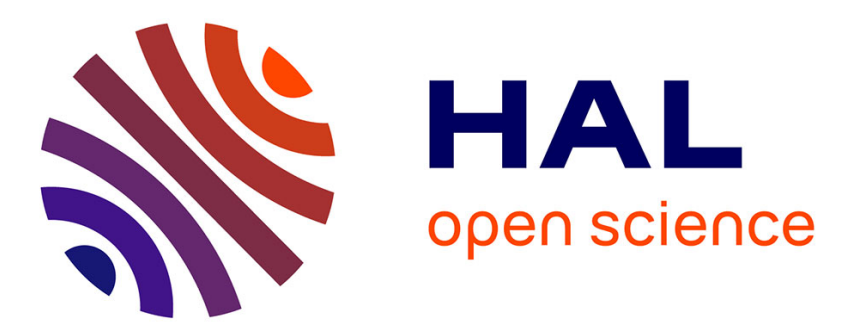

\title{
ON AN INVISCID MODEL FOR INCOMPRESSIBLE TWO-PHASE FLOWS WITH NONLOCAL INTERACTION
}

Ciprian G. Gal

\section{- To cite this version:}

Ciprian G. Gal. ON AN INVISCID MODEL FOR INCOMPRESSIBLE TWO-PHASE FLOWS WITH NONLOCAL INTERACTION. 2015. hal-01225858

\section{HAL Id: hal-01225858 \\ https://hal.science/hal-01225858}

Preprint submitted on 6 Nov 2015

HAL is a multi-disciplinary open access archive for the deposit and dissemination of scientific research documents, whether they are published or not. The documents may come from teaching and research institutions in France or abroad, or from public or private research centers.
L'archive ouverte pluridisciplinaire HAL, est destinée au dépôt et à la diffusion de documents scientifiques de niveau recherche, publiés ou non, émanant des établissements d'enseignement et de recherche français ou étrangers, des laboratoires publics ou privés. 


\title{
ON AN INVISCID MODEL FOR INCOMPRESSIBLE TWO-PHASE FLOWS WITH NONLOCAL INTERACTION
}

\author{
CIPRIAN G. GAL
}

\begin{abstract}
We consider a diffuse interface model which describes the motion of an ideal incompressible mixture of two immiscible fluids with nonlocal interaction in two-dimensional bounded domains. This model consists of the Euler equation coupled with a convective nonlocal Cahn-Hilliard equation. We establish the existence of globally defined weak solutions as well as well-posedness results for strong/classical solutions.
\end{abstract}

\section{INTRODUCTION}

In this contribution we wish to consider the following system

$$
\begin{aligned}
& \varphi_{t}+u \cdot \nabla \varphi=\Delta \mu, \mu=a \varphi-J * \varphi+F^{\prime}(\varphi), \\
& u_{t}+(u \cdot \nabla) u+\nabla \pi=\mu \nabla \varphi, \\
& \operatorname{div}(u)=0,
\end{aligned}
$$

in $\Omega \times(0, \infty)$, on a bounded domain $\Omega \subset \mathbb{R}^{2}$ with boundary $\partial \Omega$. We shall further assume the following boundary and initial conditions:

$$
\begin{array}{lll}
\nabla \mu \cdot n=0, & u \cdot n=0 & \text { on } \partial \Omega \times(0, \infty), \\
u(0)=u_{0}, & \varphi(0)=\varphi_{0} & \text { in } \Omega .
\end{array}
$$

We recall that the analogue system in which (1.2) is replaced by the Navier-Stokes equation

$$
u_{t}-\operatorname{div}(\nu(\varphi) D u)+(u \cdot \nabla) u+\nabla \pi=\mu \nabla \varphi,
$$

describes the evolution of an isothermal mixture of two incompressible and immiscible fluids through the (relative) concentration $\varphi$ of one species and the (averaged) velocity field $u$. As usual, $\mu$ is the so-called chemical potential, $J$ is a spatial-dependent interaction kernel and $J * \varphi$ stands for spatial convolution over $\Omega, a$ is defined as follows $a(x)=(J * 1)(x), F$ is a double-well potential, $\pi$ is pressure and $\nu$ is the viscosity of the two-phase fluid. This system assumes the case of matched densities for the two fluids and constant mobility. On the other hand, the system comprising of (1.6), (1.1), (1.3), subject to homogeneous Neumann and no slip boundary conditions for $\mu$ and $u$, respectively, has been analyzed recently in $[8,9,10,12,13,11]$ under various assumptions on $F, J$ and on the mobility and viscosity coefficients, respectively. We also recall that the nonlocal Cahn-Hilliard-Navier-Stokes system described earlier is a generalized version of the classical Cahn-Hilliard-Navier-Stokes system when in the place of $a \varphi-J * \varphi$ one usually finds $-\Delta \varphi$, see $[1,2,5,7,14,15,16,26,27,28]$ and references therein. For a more general family of two-phase fluid models we also refer the reader to $[18,19]$. From a modelling perspective the former term is more appropriate in the sense that it can be physically justified and rigorously derived by starting from microscopic models for lattice gases with long-range Kac potentials, see [21, 22] and 
$[4,17,20,24,25]$. However, the analysis tends to be more challenging and involved since the regularity of $\varphi$ is much lower than in the classical case. Indeed, in the case when $u \equiv 0$ in (1.1) the best one can hope for smooth solutions is that they are at most globally Holder continuous (see [17]) even when $\varphi_{0} \in C^{\infty}, F \in C^{\infty}$ and $\Omega$ is of class $C^{\infty}$ provided that $J$ is a symmetric kernel that belongs to $W_{\text {loc }}^{1,1}\left(\mathbb{R}^{2}\right)$. The latter turns out to be also optimal $[11,17]$. In particular, it is not at all expected for $\varphi$ to possess any higher order regularity in spaces like $W^{k, p}, k \geq 3$, unless $J$ is smooth and non-singular. We note that radially symmetric kernels that belong to $W_{\text {loc }}^{1,1}\left(\mathbb{R}^{2}\right)$ are not too singular at the origin and include the well-known Newtonian and Bessel potentials.

In the present article, we are interested in ideal two-phase flows (1.1)-(1.5) in domains $\Omega \subset \mathbb{R}^{2}$ with smooth boundary $\partial \Omega$ of class $C^{3}$, although this regularity can be relaxed in some places. In particular, our main goal is to prove appropriate well-posedness results for this inviscid system. From a physical perspective all twophase flows must be at least slightly viscous $(\nu=\nu(\varphi)>0)$ in the presence of a physical boundary $\partial \Omega$, and so they are properly described by the Cahn-HilliardNavier-Stokes system (1.6), (1.1), (1.3). Indeed, it is only in this case that viscous effects are essential to describe the interaction of the two-phase fluid with $\partial \Omega$ and the variation of pressure as a function of vorticity there or vice versa (see [6]). However, in the absence of physical boundaries the mathematical investigation of ideal two-phase immiscible fluid flows has some real physical relevance in turbulence modelling (see again [6]), and also in the case when the vanishing viscosity limit for incompressible flows in a domain with boundary is still to this day an important problem. We point out that our motivation also stems from some recent results of [7] which discusses the classical Cahn-Hilliard-Euler system in the case $\Omega=\mathbb{R}^{2}$ (or $\Omega$ is a bounded periodic domain) when once again in place of $a \varphi-J * \varphi$ in (1.1) one takes $-\Delta \varphi$. Indeed, in our case we are simply dealing with a parabolic equation which is of second-order for $\varphi$ instead of a fourth-order equation which was the case considered in [7] among others. Therefore, it is not straightforward to extend the results of [7] to our system (1.1)-(1.5), especially in the light of recent results proven for the nonlocal Cahn-Hilliard-Navier-Stokes system. This becomes actually more interesting when the assumptions on the potential $F$ and the interaction kernel $J$ can remain the same as in the recent work of [11], where a complete theory was developed for the full Cahn-Hilliard-Navier-Stokes system with nonlocal interaction, constant mobility and variable viscosity. We also wish to point out that the results presented in this contribution also remain true in the absence of physical boundaries when $\Omega=\mathbb{R}^{2}$ or $\Omega \subset \mathbb{R}^{2}$ is a compact manifold without boundary (cf. Remark 3.2). We leave the important question of vanishing viscosity limit to future contributions, but view the results obtained here as necessary steps in that direction.

The paper is organized as follows. In section 2 we give the main definitions for weak, strong and classical solutions and formulate statements of the main results, and in the final section 3 we provide detailed proofs of these statements.

\section{Statements of MAIn Results}

We endow $L^{p}(\Omega), W^{k, p}(\Omega)$ with the usual norms $\|\cdot\|_{L^{p}},\|\cdot\|_{W^{k, p}}$ and scalar products $(\cdot, \cdot),(\cdot, \cdot)_{W^{k, 2}}$ in $L^{2}(\Omega)$ and $W^{k, 2}(\Omega)$ respectively. With some abuse of notation we shall also use $W^{k, p}(\Omega)$ as the space of vector-valued functions. We also 
consider a closed subspace of $L^{2}(\Omega)$,

$$
H=\left\{u \in L^{2}(\Omega): \operatorname{div}(u)=0 \text { in } \Omega, u \cdot n=0 \text { on } \partial \Omega\right\} .
$$

For every $f \in\left(W^{1,2}(\Omega)\right)^{*}$ we denote by $\langle f\rangle$ the average of $f$ over $\Omega$, i.e., $\langle f\rangle=$ $|\Omega|^{-1}\langle f, 1\rangle$, where $|\Omega|$ is the Lebesgue measure of $\Omega$ and $\langle\cdot, \cdot\rangle$ denotes the usual pairing between the corresponding Banach space and its dual. Consider also the space $L_{0}^{p}(\Omega)=L^{p}(\Omega) \cap\{\varphi:\langle\varphi\rangle=0\}$. We also introduce so-called Neumann Laplacian $B_{N}=-\Delta$ which can be seen as an unbounded self-adjoint operator on $L^{2}(\Omega)$ with domain $D\left(B_{N}\right)=\left\{v \in W^{2,2}(\Omega): \nabla v \cdot n=0\right.$ on $\left.\partial \Omega\right\}$.

Beside Gagliardo-Nirenberg interpolation inequalities in two dimensions, in our proofs we shall also appeal to several fundamental inequalities. The first one is a variant of Trudinger's inequality which states for $\varphi \in W^{1,2}(\Omega)$ that

$$
\|\varphi\|_{L^{p}} \leq C p^{1 / 2}\|\varphi\|_{W^{1,2}}
$$

for any $p \in[2, \infty)$, for some constant $C>0$ independent of $p$ and $\varphi$. The second one states that any sufficiently smooth incompressible velocity $u \in H$ can be found from the vorticity $\omega=\nabla \times u$ via the Biot-Savart law

$$
u(x, t)=\int_{\Omega} B_{\Omega}(x, y) \omega(y, t) d y,
$$

where the kernel $B_{\Omega}$ is given by $B_{\Omega}(x, y)=\nabla^{\perp} G_{\Omega}(x, y)$ with $\nabla^{\perp}=\left(-\partial_{x_{2}}, \partial_{x_{1}}\right)$, $x=\left(x_{1}, x_{2}\right) \in \Omega$, and $G_{\Omega}$ is the Green function for $\Omega$, see e.g., [3, 29]. In particular, there is a constant $C>0$ independent of $p \in[2, \infty)$ such that

$$
\|\nabla u\|_{L^{p}} \leq C p\|\omega\|_{L^{p}} .
$$

Finally we also recall the following maximal regularity result for the Neumann Laplacian $B_{N}$. Specifically, it states that $\left\|B_{N}^{-1}\right\|_{L^{p} \rightarrow W^{2, p}} \leq C p$, for some $C>0$ independent of $p \in[2, \infty)$, or equivalently, for the corresponding elliptic problem $B_{N} \varphi=f \in L_{0}^{p}(\Omega)$, we have the estimate

$$
\|\varphi\|_{W^{2, p}} \leq C p\|f\|_{L^{p}} .
$$

We now formulate the notion of a globally defined weak solution for the inviscid problem (1.1)-(1.5).

Definition 2.1. Let $u_{0} \in V:=W^{1,2}(\Omega) \cap H, \varphi_{0} \in W:=W^{1,2}(\Omega) \cap L^{\infty}(\Omega)$ and $T>0$ be given. We say that $(u, \varphi)$ is a weak solution to problem (1.1)-(1.5) corresponding to a given $\left(u_{0}, \varphi_{0}\right)$ if the following hold:

- The functions $u, \varphi$ satisfy

$$
\begin{aligned}
& u \in L^{\infty}(0, T ; V), \partial_{t} u \in L^{2}\left(0, T ; V^{*}\right), \\
& \varphi \in L^{\infty}(0, T ; W) \cap L^{2}\left(0, T ; W^{2,2}(\Omega)\right) \cap W^{1,2}\left(0, T ; L^{2}(\Omega)\right), \\
& \mu \in L^{\infty}(0, T ; W) \cap L^{2}\left(0, T ; W^{2,2}(\Omega)\right) .
\end{aligned}
$$

- For every $\psi \in H^{1}(\Omega)$, every $v \in V$ and for almost any $t \in(0, T)$ we have

$$
\begin{aligned}
& \left(\partial_{t} \varphi, \psi\right)+(u \varphi, \nabla \psi)=(\nabla \mu, \nabla \psi), \\
& \left\langle\partial_{t} u, v\right\rangle+\langle u \cdot \nabla u, v\rangle=(\mu \nabla \varphi, v),
\end{aligned}
$$

such that $\mu=a(x) \varphi-J * \varphi+F^{\prime}(\varphi)$, a.e. in $\Omega \times(0, T)$.

- The initial conditions $u(0)=u_{0}, \varphi(0)=\varphi_{0}$ hold in following sense: $u(t) \rightarrow$ $u_{0}$ as $t \rightarrow 0$ in $H$-sense, and $\varphi(t) \rightarrow \varphi_{0}$ as $t \rightarrow 0$ in the $L^{2}(\Omega)$-sense. 
We also define what we mean by a strong/classical solution to the Cahn-HilliardEuler system with nonlocal interaction.

Definition 2.2. Let $u_{0} \in V, \omega_{0}=\nabla \times u_{0} \in L^{q}(\Omega)$, for some $q \in(2, \infty)$ and $\varphi \in W^{2,2}(\Omega)$.

- We say that $(u, \varphi)$ is a strong solution of (1.1)-(1.5) if it is a weak solution in the sense of Definition 2.1 and in addition,

$$
\begin{aligned}
\partial_{t} u & \in L^{\infty}\left(0, T ; L^{q}(\Omega)\right), \omega=\nabla \times u \in L^{\infty}\left(0, T ; L^{q}(\Omega)\right), \\
\varphi & \in L^{\infty}\left(0, T ; W^{2,2}(\Omega)\right) \cap W^{1, \infty}\left(0, T ; L^{2}(\Omega)\right), \\
\varphi & \in W^{1,2}(\Omega \times(0, T)) \cap C^{\beta, \beta / 2}(\bar{\Omega} \times([0, T])), \beta \in(0,1), \\
\mu & \in L^{\infty}\left(0, T ; D\left(B_{N}\right)\right) \cap W^{1, \infty}\left(0, T ; L^{2}(\Omega)\right) .
\end{aligned}
$$

- We say that $(u, \varphi)$ is a classical solution if it is a strong solution that satisfies

$$
u \in L^{\infty}\left(0, T ; W^{3,2}(\Omega) \cap H\right), \varphi \in L^{\infty}\left(0, T ; W^{2, p}(\Omega)\right),
$$

provided that in addition $u_{0} \in W^{3,2}(\Omega) \cap H$ and $\varphi_{0} \in W^{2, p}(\Omega)$ for some $p \in(2, \infty)$.

Our assumptions on $F, J$ remain essentially the same as in $[8,9,12,11,17]$, and actually we can require much less than there.

(H1) $J \in W_{\text {loc }}^{1,1}\left(\mathbb{R}^{d}\right), \quad J(x)=J(-x), \quad a \geq 0$ a.e. in $\Omega$.

(H2) $F$ is a regular potential which belongs to $C^{2}(\mathbb{R}, \mathbb{R})$ and there exists $c_{0}, c_{1}>0$, $c_{2} \geq 0$ such that

$$
F^{\prime \prime}(s)+a(x) \geq c_{0}, F(s) \geq c_{1} s^{2}-c_{2},
$$

for all $s \in \mathbb{R}$, a.e. $x \in \Omega$.

The first main result is on the weak solvability of problem (1.1)-(1.5).

Theorem 2.3. Let the assumptions (H1)-(H2) be satisfied and assume $u_{0} \in V:=$ $W^{1,2}(\Omega) \cap H, \varphi_{0} \in W:=H^{1}(\Omega) \cap L^{\infty}(\Omega)$. Then there exists at least one globally defined weak solution in the sense of Definition 2.1.

In order to provide the final results we need to introduce an additional assumption on the kernel $J$ exactly as in [11].

Definition 2.4. A kernel $J \in W_{\text {loc }}^{1,1}\left(\mathbb{R}^{2}\right)$ satisfying (H1) is said to be admissible if either $J \in W^{2,1}\left(B_{\delta}\right), \delta \sim \operatorname{diam}(\Omega)$, or the following conditions are satisfied:

(A1) $J \in C^{3}\left(\mathbb{R}^{2} \backslash\{0\}\right)$;

(A2) $J$ is radially symmetric, $J(x)=\rho(|x|)$ and $\rho$ is non-increasing;

(A3) $\rho^{\prime \prime}(r)$ and $\rho^{\prime}(r) / r$ are monotone on $\left(0, r_{0}\right)$ for some $r_{0}>0$;

(A4) $\left|D^{3} J(x)\right| \leq C_{\sharp}|x|^{-3}$ for some $C_{\sharp}>0$.

We recall that the Newtonian and Bessel potentials are admissible, and in particular the following estimate holds for a kernel $J$ satisfying (A1)-(A4):

$$
\|\nabla(\nabla J * \varphi)\|_{p} \leq C p\|\varphi\|_{L^{p}},
$$

for some $C>0$ independent of $p \in[2, \infty)$ and $\varphi$ (see, e.g., [11]).

Theorem 2.5. Let $(u, \varphi)$ be a weak solution in the sense of Definition 2.1 with bounded vorticity $\omega=\nabla \times u \in L^{\infty}\left(0, T ; L^{\infty}(\Omega)\right)$. Then problem (1.1)-(1.5) has at most one such weak solution. 
Theorem 2.6. Assume $J$ is admissible in the sense of Definition 2.4. Then there exists at least one strong solution and a unique classical solution in the sense of Definition 2.2.

\section{Proofs of the main Results}

In this section we provide detailed proofs of the statements of Theorem 2.3 and Theorem 2.6. Throughout this section, $C \geq 0$ will denote a generic constant whose further dependence on certain quantities will be specified on occurrence. The value of the constant can change even within the same line. Furthermore, we introduce the notation $a \lesssim b$ to mean that there exists a constant $C>0$ such that $a \leq C b$. This notation will be used when the explicit value of $C$ is irrelevant or tedious to write down. We divide our program into two parts: we first provide formal estimates leading to the required estimates in the statements of Theorems 2.3, 2.6, and then at the end we briefly provide the details of the approximation scheme and fixed point arguments that are necessary to construct smooth solutions on which the formal estimates can be ultimately performed. The passage to the limit in the smooth solutions will be standard owing to uniform estimates obtained in the previous steps, and therefore will be altogether omitted.

We begin with a basic estimate for the energy functional associated with problem (1.1)-(1.5). Let

$$
E(t):=\frac{1}{2}\|u(t)\|_{L^{2}}^{2}+\frac{1}{4} \int_{\Omega} \int_{\Omega} J(x-y)(\varphi(x, t)-\varphi(y, t))^{2} d y d x+\int_{\Omega} F(\varphi(t)) d x
$$

Proposition 3.1. Let $u_{0} \in H$ and $\varphi_{0} \in L^{\infty}(\Omega)$ and assume (H1)-(H2). Then there exists a constant $C>0$ independent of time, $T>0$, depending on $\left(u_{0}, \varphi_{0}\right)$, such that

$$
\sup _{t \in[0, T]} E(t)+\int_{0}^{T} \int_{\Omega}|\nabla \mu(x, t)| d x d t \leq C .
$$

Moreover, by virtue of (3.1) it follows that

$$
\begin{aligned}
\varphi & \in L^{\infty}\left(0, T ; L^{\infty}(\Omega)\right) \cap L^{2}\left(0, T ; H^{1}(\Omega)\right), \\
\mu & \in L^{2}\left(0, T ; H^{1}(\Omega)\right) \cap L^{\infty}\left(0, T ; L^{\infty}(\Omega)\right), \\
\partial_{t} \varphi & \in L^{2}\left(0, T ;\left(H^{1}(\Omega)\right)^{*}\right),
\end{aligned}
$$

uniformly in time $t \in(0, T)$, for any $T>0$.

Proof. We multiply the first and second equations of (1.1) scalarly by $\mu$ and $\partial_{t} \varphi$, respectively, then equation (1.2) scalarly by $u$, and integrate by parts using the fact that $u$ is divergent free, $u \cdot n=0$ and $\nabla \mu \cdot n=0$ on $\partial \Omega$, to obtain

$$
\frac{d}{d t} E(t)+\int_{\Omega}|\nabla \mu(t)| d x=0, t \in(0, T) .
$$

We also refer the reader to $[8,12,11]$ for further details concerning this identity. In particular, integrating the foregoing relation over $(0, t)$ with $t \in(0, T)$ gives

$$
\sup _{t \in[0, T]} E(t)+\int_{0}^{T} \int_{\Omega}|\nabla \mu(x, t)| d x d t \leq E(0),
$$

from which (3.1) follows. Indeed, for $\varphi_{0} \in L^{\infty}(\Omega), u_{0} \in H$ and $J \in L_{\text {loc }}^{1}\left(\mathbb{R}^{2}\right)$ we have $E(0) \leq C\left(u_{0}, \varphi_{0},\|J\|_{L^{1}}\right)$. Next, we observe that owing to assumption 
(H2), we can find two positive constants $c_{1}>, c_{2} \geq 0$ such that $F(s) \geq c_{1} s^{2}-c_{2}$, for all $s \in \mathbb{R}$. Hence, from (3.5) we clearly have $\varphi \in L^{\infty}\left(0, T ; L^{2}(\Omega)\right)$ and by virtue of $\left[4\right.$, Theorem 2.1] it also follows that $\varphi \in L^{\infty}\left(0, T ; L^{\infty}(\Omega)\right)$ uniformly in time $t \in(0, T)$ and $T>0$, in dependance only of $E(0)<\infty$. Furthermore, since $\mu=a(x) \varphi-J * \varphi+F^{\prime}(\varphi)$ we have owing to the first of assumption (H2) and a standard computation (see, e.g., $[11,(4.23)]$ ) that for any $p \in(1, \infty)$,

$$
\|\nabla \varphi\|_{L^{p}} \leq\|\nabla \mu\|_{L^{p}}+C\left(R,\|J\|_{W^{1,1}}, p, c_{0}\right),
$$

for $R=R(E(0))>0$ such that $\|\varphi\|_{L^{\infty}\left(0, T ; L^{\infty}(\Omega)\right)} \leq R$. By (3.5) and (3.6), the second assertion of (3.2) follows immediately as well. It is also clear that (3.3) holds by definition in light of the first of (3.2) and the fact that $J \in L^{1}, a \in L^{\infty}$. Finally, (3.4) is also verified by a comparison argument in (1.1) in light of (3.2)-(3.3) and the fact that $u \in L^{\infty}(0, T ; H)$, which is a consequence of (3.1).

We aim to deduce higher-order estimates for the solution of (1.1)-(1.5). To this end, we shall apply the curl operator to equation (1.2) to eliminate the pressure term $\pi$. We obtain an equation for the vorticity $\omega=\nabla \times u=\partial_{x_{1}} u_{2}-\partial_{x_{2}} u_{1}$ associated with the velocity $u=\left(u_{1}, u_{2}\right)$, as follows:

$$
\partial_{t} \omega+u \cdot \nabla \omega=-\nabla \mu \cdot \nabla^{\perp} \varphi, \text { in } \Omega \times(0, T),
$$

where $u$ is still subject to the boundary and initial conditions of (1.4)-(1.5). We also rewrite the equation (1.1) for the order parameter $\varphi$, as follows:

$$
\partial_{t} \varphi+b^{-1}(u \cdot \nabla \mu)+b^{-1} u \cdot(\nabla J * \varphi-(\nabla a) \varphi)=-B_{N} \mu, \text { in } \Omega \times(0, T),
$$

where $b(x, \varphi):=a(x)+F^{\prime \prime}(\varphi) \geq c_{0}$ by $(\mathrm{H} 2)$, as well as $b \in L^{\infty}\left(0, T ; L^{\infty}(\Omega)\right)$ provided that $\varphi \in L^{\infty}\left(0, T ; L^{\infty}(\Omega)\right)$ (this was already established earlier in (3.2)).

Theorem 3.2. Let $u_{0} \in V=W^{1,2}(\Omega) \cap H$ and $\varphi_{0} \in W=L^{\infty}(\Omega) \cap W^{1,2}(\Omega)$. Assume that $J$ is admissible in the sense of Definition 2.4. Then any smooth solution of (1.1)-(1.5) satisfies

$$
\begin{aligned}
\varphi & \in L^{\infty}(0, T ; W) \cap L^{2}\left(0, T ; W^{2,2}(\Omega)\right), \\
\mu & \in L^{2}\left(0, T ; D\left(B_{N}\right)\right) \cap L^{\infty}\left(0, T ; W^{1,2}(\Omega)\right), \\
\partial_{t} \varphi & \in L^{2}\left(0, T ; L^{2}(\Omega)\right), u \in L^{\infty}(0, T ; V),
\end{aligned}
$$

uniformly in time $t \in(0, T)$, for any $T>0$. In addition if $\varphi_{0} \in C^{\alpha}(\bar{\Omega}), \alpha \in(0,1)$ then

$$
\varphi \in C^{\beta, \beta / 2}(\bar{\Omega} \times([0, T])), \text { for some } \beta \in(0,1) .
$$

Proof. In order to derive the desired regularity in (3.9)-(3.10) it suffices to establish the following uniform bounds

$$
\mu \in L^{\infty}\left(0, T ; W^{1,2}(\Omega)\right), u \in L^{\infty}(0, T ; V) .
$$

In the proof of [11, Theorem 5] this was done by deriving the first bound of (3.13) using only (3.8) and some a priori information based on which the velocity $u \in L^{2}\left(0, T ; W^{1,2}(\Omega)\right)$. We recall that the latter regularity is readily available for the Navier-Stokes equation (1.6) by the energy estimate performed earlier in Proposition 3.1 (cf. e.g., [8]) whereas in the case of the Euler equation (1.2) much less is true, see (3.1). On the other hand, our argument here makes also use of the vorticity equation (3.7), which exploited in unison with (3.8) can produce the 
required bounds in (3.13). Therefore, we test the nonlocal Cahn-Hilliard equation (3.8) by $\partial_{t} \mu=b(x, \varphi) \partial_{t} \varphi-J * \partial_{t} \varphi$ in $L^{2}(\Omega)$ to deduce

$$
\int_{\Omega} \partial_{t} \varphi \partial_{t} \mu+\int_{\Omega}\left(b^{-1}(u \cdot \nabla \mu)+b^{-1} u \cdot(\nabla J * \varphi-(\nabla a) \varphi)\right) \partial_{t} \mu+\frac{1}{2} \frac{d}{d t}\|\nabla \mu\|_{L^{2}}^{2}=0 .
$$

To estimate the first two integral terms on the left-hand side of (3.14) we can use the same arguments of $[11,(4.17)-(4.21)]$ to derive

$$
\begin{aligned}
& \frac{d}{d t}\|\nabla \mu\|_{L^{2}}^{2}+\frac{c_{0}}{2}\left(\left\|\partial_{t} \varphi\right\|_{L^{2}}^{2}+\frac{1}{2}\left\|B_{N} \mu\right\|_{L^{2}}^{2}\right) \\
& \leq C_{c_{0}, J, \epsilon}(R)\left(\|u\|_{L^{2}}^{2}\|\nabla u\|_{L^{2}}^{2}\right)\|\nabla \mu\|_{L^{2}}^{2}+c\|J\|_{W^{1,1}}^{2}\left\|\partial_{t} \varphi\right\|_{\left(H^{1}\right)^{*}}^{2} \\
& +C_{c_{0}, J}(R)\|u\|_{L^{2}}^{2}+2 \epsilon\left(\left\|B_{N} \mu\right\|_{L^{2}}^{2}+\|\mu\|_{L^{2}}^{2}\right)
\end{aligned}
$$

for any $\epsilon>0$. Let us now choose a sufficiently small $\epsilon \leq c_{0} / 8$ in order to absorb the $L^{2}$-norm of $B_{N} \mu$ into the left-hand side and observe that $\mu \in L^{\infty}(\Omega \times(0, T))$ since $\varphi$ is bounded. Furthermore, we shall exploit the inequality (2.2). Thus, we find

$$
\begin{aligned}
& \frac{d}{d t}\|\nabla \mu\|_{L^{2}}^{2}+\epsilon_{0}\left(\left\|\partial_{t} \varphi\right\|_{L^{2}}^{2}+\left\|B_{N} \mu\right\|_{L^{2}}^{2}\right) \\
& \leq C\left(\|u\|_{L^{2}}^{2}+\|\mu\|_{L^{2}}^{2}+\left\|\partial_{t} \varphi\right\|_{\left(H^{1}\right)^{*}}^{2}\right)+C\left(\|u\|_{L^{2}}^{2}\|\nabla \mu\|_{L^{2}}^{2}\right)\|\omega\|_{L^{2}}^{2},
\end{aligned}
$$

for some $\epsilon_{0}>0$ and $C=C\left(c_{0}, J, e_{0}, R\right)>0$. We now test the vorticity equation (3.7) in $L^{2}(\Omega)$ by $\omega$, use the boundary condition $u \cdot n=0$ on $\partial \Omega$ and the fact that $u$ is divergent free, such that

$$
\frac{d}{d t}\|\omega\|_{L^{2}}^{2}=-\left(\nabla \mu \cdot \nabla^{\perp} \varphi, \omega\right) \leq\left\|\nabla \mu \cdot \nabla^{\perp} \varphi\right\|_{L^{2}}\|\omega\|_{L^{2}} .
$$

Since $\left\|\nabla^{\perp} \varphi\right\|_{L^{p}}=\|\nabla \varphi\|_{L^{p}}$ and $\nabla^{\perp} \varphi=b^{-1} \nabla^{\perp} \mu+b^{-1}\left(\nabla^{\perp} J * \varphi-\left(\nabla^{\perp} a\right) \varphi\right)$, we have

$$
\begin{aligned}
& \left\|\nabla \mu \cdot \nabla^{\perp} \varphi\right\|_{L^{2}}\|\omega\|_{L^{2}} \\
& \leq\|\nabla \mu\|_{L^{4}}\left\|\nabla^{\perp} \varphi\right\|_{L^{4}}\|\omega\|_{L^{2}} \\
& \leq C\left(c_{0}\right)\left(\|\nabla \mu\|_{L^{4}}^{2}+\left\|\nabla^{\perp} J * \varphi\right\|_{L^{4}}\|\nabla \mu\|_{L^{4}}+\left\|\nabla^{\perp} a\right\|_{L^{4}}\|\nabla \mu\|_{L^{4}}\right)\|\omega\|_{L^{2}} \\
& \leq C\left(c_{0},\|J\|_{W^{1,1}}, R\right)\|\nabla \mu\|_{L^{2}}\left(\left\|B_{N} \mu\right\|_{L^{2}}+\|\mu\|_{L^{2}}\right)\|\omega\|_{L^{2}} \\
& \leq C\left(c_{0}, \epsilon,\|J\|_{W^{1,1}}, R\right)\|\nabla \mu\|_{L^{2}}^{2}\|\omega\|_{L^{2}}^{2}+\epsilon\left(\left\|B_{N} \mu\right\|_{L^{2}}^{2}+\|\mu\|_{L^{2}}^{2}\right)
\end{aligned}
$$

for any $\epsilon>0$, since $\nabla^{\perp} a \in L^{\infty}$ owing to $J \in W^{1,1}$, provided that $\|\varphi\|_{L^{\infty}\left(0, T ; L^{\infty}(\Omega)\right)} \leq$ $R$. Inserting (3.18) into the right-hand side of (3.17) and adding the resulting inequality to (3.16), and selecting a sufficiently small $\epsilon<\epsilon_{0}$, we infer

$$
\begin{aligned}
& \frac{d}{d t}\left(\|\nabla \mu\|_{L^{2}}^{2}+\|\omega\|_{L^{2}}^{2}\right)+\bar{\epsilon}_{0}\left(\left\|\partial_{t} \varphi\right\|_{L^{2}}^{2}+\left\|B_{N} \mu\right\|_{L^{2}}^{2}\right) \\
& \leq C\left(\|u\|_{L^{2}}^{2}+\|\mu\|_{L^{2}}^{2}+\left\|\partial_{t} \varphi\right\|_{\left(H^{1}\right)^{*}}^{2}\right)+C\left(\|u\|_{L^{2}}^{2}+1\right)\|\nabla \mu\|_{L^{2}}^{2}\|\omega\|_{L^{2}}^{2},
\end{aligned}
$$

for some $\bar{\epsilon}_{0}>0$ and $C=C\left(c_{0}, \epsilon,\|J\|_{W^{1,1}}, R, \bar{\epsilon}_{0}\right)>0$. Since $\varphi$ is bounded, and so is $\mu$, we observe that by virtue of the uniform bounds established in the foregoing Proposition 3.1, we have by application of Gronwal"s inequality that

$$
\begin{aligned}
\partial_{t} \varphi & \in L^{2}\left(0, T ; L^{2}(\Omega)\right), u \in L^{\infty}(0, T ; V), \\
\mu & \in L^{\infty}\left(0, T ; W^{1,2}(\Omega)\right) \cap L^{2}\left(0, D\left(B_{N}\right)\right),
\end{aligned}
$$


by means of the Biot-Savart inequality (2.2) (indeed, $\varphi_{0} \in W$ implies that $\mu_{0} \in W$ by the definition of the chemical potential). The first of (3.21) together with (3.6) implies in particular

$$
\varphi \in L^{\infty}\left(0, T ; W^{1,2}(\Omega)\right) .
$$

Furthermore, the continuous embedding

$$
L^{\infty}\left(0, T ; W^{1,2}(\Omega)\right) \cap L^{2}\left(0, D\left(B_{N}\right)\right) \subset L^{4}\left(0, T ; W^{1,4}(\Omega)\right)
$$

allows us to conclude from (3.21) and (3.6) that

$$
\mu, \varphi \in L^{4}\left(0, T ; W^{1,4}(\Omega)\right) .
$$

As in the proof of [11, Theorem 5] we now control the $H^{2}$-norm of $\varphi$ (or at least the $L^{2}$-norm of the second derivatives $\left.\partial_{i j}^{2} \varphi:=\frac{\partial^{2} \varphi}{\partial x_{i} \partial x_{j}}\right)$ in terms of the $H^{2}$-norm of $\mu$ and (3.23). To this aim apply the second derivative operator $\partial_{i j}^{2}$ to the second of (1.1, multiply the resulting identity by $\partial_{i j}^{2} \varphi$ and integrate on $\Omega$. This entails

$$
\begin{aligned}
\left(\partial_{i j}^{2} \mu, \partial_{i j}^{2} \varphi\right) & =\left(a+F^{\prime \prime}(\varphi),\left(\partial_{i j}^{2} \varphi\right)^{2}\right)+\left(\partial_{i} a \partial_{j} \varphi+\partial_{j} a \partial_{i} \varphi, \partial_{i j}^{2} \varphi\right) \\
& +\left(\varphi \partial_{i j}^{2} a-\partial_{i}\left(\partial_{j} J * \varphi\right), \partial_{i j}^{2} \varphi\right)+\left(F^{\prime \prime \prime}(\varphi) \partial_{i} \varphi \partial_{j} \varphi, \partial_{i j}^{2} \varphi\right) .
\end{aligned}
$$

This identity and the first of assumption (H2) yields

$$
\begin{aligned}
& c_{0}\left\|\partial_{i j}^{2} \varphi\right\|^{2} \leq c\left\|\partial_{i j}^{2} \mu\right\|^{2} \\
& +c\left(\|\nabla a\|_{L^{\infty}}^{2}+Q(R)\right)\|\nabla \varphi\|^{2}+Q(R)\left\|\partial_{i j}^{2} a\right\|^{2} \\
& +\left\|\partial_{i}\left(\partial_{j} J * \varphi\right)\right\|^{2}+Q(R)\|\nabla \varphi\|_{L^{4}}^{4},
\end{aligned}
$$

and an estimate like this still holds if $\left\|\partial_{i j}^{2} \varphi\right\|$ and $\left\|\partial_{i j}^{2} \mu\right\|$ are replaced by $\|\varphi\|_{H^{2}}$ and $\|\mu\|_{H^{2}}$, respectively. Thus, recalling (3.20)-(3.23) and using the fact that $J \in$ $W^{2,1}\left(B_{\delta}\right)$ or $J$ is admissible (i.e., (2.14) holds), from (3.25) we easily get

$$
\varphi \in L^{2}\left(0, T ; H^{2}(\Omega)\right) \text {. }
$$

Collecting (3.20)-(3.22) and (3.26) the desired properties in (3.9)-(3.11) are then verified. For the final regularity in (3.12), we can now apply the result of [11, Lemma $2]$ to conclude owing to the fact that $u \in L^{\infty}(0, T ; V) \subset L^{4}\left(0, T ; L^{4}(\Omega)\right)$.

Lemma 3.3. Let the assumptions of Theorem 3.2 be satisfied and suppose in addition that $\omega_{0}=\nabla \times u_{0} \in L^{q}(\Omega)$, for some given $q \in(2, \infty)$. Then the solution $(u, \varphi)$ satisfies

$$
\omega=\nabla \times u \in L^{\infty}\left(0, T ; L^{q}(\Omega)\right), \partial_{t} u \in L^{\infty}\left(0, T ; L^{2}(\Omega)\right) .
$$

In particular, the following explicit estimate holds:

$$
\sup _{t \in(0, T)}\|\omega(t)\|_{L^{q}} \leq\left\|\omega_{0}\right\|_{L^{q}}+C_{T} q^{1 / 2},
$$

for some $C_{T}>0$ independent of $u, \varphi, q \in(2, \infty)$ and time $t \in(0, T)$.

Proof. We test the vorticity equation $(3.7)$ in $L^{2}(\Omega)$ by $|\omega|^{q-2} \omega$, use the boundary condition $u \cdot n=0$ on $\partial \Omega$ and $u$ is divergent free, to deduce

$$
\begin{aligned}
\frac{1}{q} \frac{d}{d t}\|\omega\|_{L^{q}}^{q} & =-\left(\nabla \mu \cdot \nabla^{\perp} \varphi,|\omega|^{q-2} \omega\right) \\
& =-\int_{\Omega}|\omega|^{q-2} \omega b^{-1}\left(\nabla \mu \cdot \nabla^{\perp} J * \varphi-\nabla \mu \cdot\left(\nabla^{\perp} a\right) \varphi\right) \\
& \leq C\left(c_{0}\right)\|\omega\|_{L^{q}}^{q-1}\left(\left\|\nabla \mu \cdot \nabla^{\perp} J * \varphi\right\|_{L^{q}}+\left\|\nabla \mu \cdot\left(\nabla^{\perp} a\right) \varphi\right\|_{L^{q}}\right)
\end{aligned}
$$




$$
\leq C\left(c_{0}, R,\|J\|_{W^{1,1}}\right)\|\omega\|_{L^{q}}^{q-1}\|\nabla \mu\|_{L^{q}},
$$

owing once again to the fact that $\nabla^{\perp} \varphi=b^{-1} \nabla^{\perp} \mu+b^{-1}\left(\nabla^{\perp} J * \varphi-\left(\nabla^{\perp} a\right) \varphi\right)$ and $c_{0} \leq b \leq C(R, J)$ (also note that $\nabla \mu \cdot \nabla^{\perp} \mu=0$ ). In particular, the foregoing estimate yields

$$
\frac{d}{d t}\|\omega\|_{L^{q}} \leq C\left(c_{0}, R,\|J\|_{W^{1,1}}\right)\|\nabla \mu\|_{L^{q}} \leq C q^{1 / 2}\|\mu\|_{H^{2}}
$$

where in the last inequality we have exploited the Trudinger inequality (2.1). The constant $C>0$ in (3.30) is clearly independent of $q \in[2, \infty)$. Integrating (3.30) over time and exploiting (3.21) to control the $L^{1}\left(0, T ; H^{2}(\Omega)\right)$-norm of $\mu$ yields the desired conclusion in (3.28). Thus, the first of (3.27) has been verified. It is left to check the second of (3.27). To this end, we test the Euler equation (1.2) in $H$ by $\partial_{t} u$ to find

$$
\begin{aligned}
\left\|\partial_{t} u\right\|_{L^{2}}^{2} & =-\int_{\Omega}(u \cdot \nabla u) \partial_{t} u+\int_{\Omega}(\mu \nabla \varphi) \partial_{t} u \\
& \leq\left\|\partial_{t} u\right\|_{L^{2}}\left(\|u \cdot \nabla u\|_{L^{2}}+\|\mu \nabla \varphi\|_{L^{2}}\right)
\end{aligned}
$$

using the fact that $u \cdot n=0$ on $\partial \Omega$ (which implies that $\partial_{t} u \cdot n=\partial_{t}(u \cdot n)=0$ on $\partial \Omega)$ as well as $\partial_{t}(\nabla \cdot u)=0$. More precisely, in view of (2.2) and the continuous embedding $W^{1, q} \subset L^{\infty}$ for $q>2$ it holds

$$
\begin{aligned}
\left\|\partial_{t} u\right\|_{L^{2}} & \leq\|u \cdot \nabla u\|_{L^{2}}+\|\mu \nabla \varphi\|_{L^{2}} \\
& \leq\|u\|_{L^{\infty}}\|\nabla u\|_{L^{2}}+\|\mu\|_{L^{\infty}}\|\varphi\|_{W^{1,2}} \\
& \leq C q\|\omega\|_{L^{q}}\|u\|_{V}+C(R, J)\|\varphi\|_{W^{1,2}} .
\end{aligned}
$$

Thus, from (3.22), (3.28) and the second of (3.20) we obtain the desired conclusion in $(3.27)$.

The previous estimates can be used to derive a sufficient condition so that our problem (1.1)-(1.5) has bounded vorticity.

Corollary 3.4. Let the assumptions of Lemma 3.3 be satisfied and further assume that $T>0$ is such that

$$
\int_{0}^{T}\|\nabla \mu(t)\|_{L^{\infty}} d t \leq C_{T}
$$

Then $\omega \in L^{\infty}\left(0, T ; L^{\infty}(\Omega)\right)$ provided that in addition $\omega_{0} \in L^{\infty}(\Omega)$.

Proof. The proof is immediate owing to the first inequality of (3.30) that can now be integrated in time. The procedure yields an inequality in which the passage to the limit as $q \rightarrow \infty$ can be easily performed, owing to the fact that the constant on the right-hand side is independent of $q>2$.

Remark 3.1. In light of Lemma 3.3 note that we also have $\pi \in L^{\infty}\left(0, T ; H^{1}(\Omega) / \mathbb{R}\right)$ by comparison in (1.2). Moreover, (3.32) can be also stated equivalently in terms of

$$
\int_{0}^{T}\|\nabla \varphi(t)\|_{L^{\infty}} d t \leq C_{T}
$$

Lemma 3.5. Let the assumptions of Lemma 3.3 hold. Then the solution $(u, \varphi)$ satisfies

$$
\varphi \in L^{\infty}\left(0, T ; W^{2,2}(\Omega)\right) \cap W^{1, \infty}\left(0, T ; L^{2}(\Omega)\right)
$$




$$
\mu \in L^{\infty}\left(0, T ; D\left(B_{N}\right)\right) \cap W^{1,2}\left(0, T ; L^{2}(\Omega)\right)
$$

provided that in addition $\varphi_{0} \in W^{2,2}(\Omega)$. It follows that

$$
\partial_{t} u \in L^{\infty}\left(0, T ; L^{q}(\Omega)\right),
$$

for any $q \in(2, \infty)$ such that $\omega_{0}=\nabla \times u_{0} \in L^{q}(\Omega)$.

Proof. Recall that (3.27) holds. The proof of the first part of this lemma follows immediately from that of [12, Theorem 2] where a bound $u \in L^{2}\left(0, T ; W^{2,2}(\Omega) \cap H\right)$ was used on the velocity. In fact, estimating in a more accurate way we can replace this bound in terms of that $\omega \in L^{\infty}\left(0, T ; L^{q}(\Omega)\right)$ for the vorticity for some $q \in(2, \infty)$. Indeed the latter yields

$$
u \in L^{\infty}\left(0, T ; W^{1, q}(\Omega)\right) \subset L^{\infty}\left(0, T ; L^{\infty}(\Omega)\right)
$$

and so the same arguments in Step 2 of [12, Theorem 2] work with no essential modifications. It remains to show (3.35); this turns out to be an improved version of the second of (3.27). Using the usual Hedge decomposition of $L^{2}(\Omega)$, we apply the Leray projector $P: L^{2}(\Omega) \rightarrow H$, which is also bounded on $L^{p}(\Omega)$, to equation (1.2) giving

$$
\partial_{t} u+P(u \cdot \nabla u)=P(\mu \nabla \varphi), \text { in } \Omega \times(0, T) .
$$

We then test (3.36) in $L^{2}(\Omega)$ by $\left|\partial_{t} u\right|^{p-2} \partial_{t} u$ for some $p \in(2, \infty)$, to deduce

$$
\begin{aligned}
\left\|\partial_{t} u\right\|_{L^{p}}^{p} & =-\left(P(u \cdot \nabla u),\left|\partial_{t} u\right|^{p-2} \partial_{t} u\right)+\left(P(\mu \nabla \varphi),\left|\partial_{t} u\right|^{p-2} \partial_{t} u\right) \\
& \leq\left(\|u \cdot \nabla u\|_{L^{p}}+\|\mu \nabla \varphi\|_{L^{p}}\right)\left\|\partial_{t} u\right\|_{L^{p}}^{p-1},
\end{aligned}
$$

from which we obtain

$$
\begin{aligned}
\left\|\partial_{t} u\right\|_{L^{p}} & \leq\|u \cdot \nabla u\|_{L^{p}}+\|\mu \nabla \varphi\|_{L^{p}} \\
& \leq C p\|u\|_{L^{\infty}}\|\omega\|_{L^{p}}+C p^{1 / 2}\|\mu\|_{L^{\infty}}\left(\|\nabla \varphi\|_{H^{1}}\right) \\
& \leq C p\|\omega\|_{L^{q}}\|\omega\|_{L^{p}}+C(R) p^{1 / 2}\|\varphi\|_{H^{2}} \\
& \leq C(R) p\left(\|\omega\|_{L^{q}}^{2}+\|\varphi\|_{H^{2}}\right)
\end{aligned}
$$

provided that $p \leq q$. Here we have exploited the Trudinger inequality (2.1) and the Biot-Savart inequality (2.2). Thus, (3.35) also follows in view of the regularity (3.33) and the first of (3.27).

It remains to deduce a higher-order estimate for the order parameter $\varphi$, which will allow us to obtain the control of (3.32). This will be performed by an iterative argument on the nonlocal Cahn-Hilliard equation (1.1) to derive a regularity result on $\partial_{t} \varphi \in L^{\infty}\left(0, T ; L^{p}(\Omega)\right)$ for any $p \in(2, \infty)$. Indeed, by the control of (3.33), (3.27) we can see that

$$
B_{N} \mu=f:=-\partial_{t} \varphi-u \cdot \nabla \varphi \in L^{\infty}\left(0, T ; L^{p}(\Omega)\right),
$$

for any $p \in(2, \infty)$; this result together with the maximal regularity of the Neumann Laplacian yields from (2.3) that

$$
\mu \in L^{\infty}\left(0, T ; W^{2, p}(\Omega)\right), \text { for any } p \in(2, \infty) .
$$

In particular, the continuous embedding $W^{2, p} \subset W^{1, \infty}$ yields from (3.38) the desired control in (3.32) so that Corollary 3.4 can be indeed verified. However, let us mention that in the Cahn-Hilliard equation (1.1) we have

$$
\partial_{t} \varphi(0)=-u_{0} \cdot \nabla \varphi_{0}-B_{N} \mu(0) \in L^{p}(\Omega)
$$


if and only if $\mu(0) \in W^{2, p}(\Omega)$ since $u_{0} \cdot \nabla \varphi_{0} \in L^{p}(\Omega)$, for any $p \in(2, \infty)$ owing to the fact that $\omega_{0} \in L^{\infty}(\Omega)$ and $\varphi_{0} \in W^{2,2}(\Omega)$. By definition of the chemical potential $\mu$ (see the second of (1.1)), from the identity (3.24) we have

$$
\begin{aligned}
\partial_{i j}^{2} \mu & =\left(a+F^{\prime \prime}(\varphi)\right) \partial_{i j}^{2} \varphi+\partial_{i} a \partial_{j} \varphi+\partial_{j} a \partial_{i} \varphi \\
& +\varphi \partial_{i j}^{2} a-\partial_{i}\left(\partial_{j} J * \varphi\right)+F^{\prime \prime \prime}(\varphi) \partial_{i} \varphi \partial_{j} \varphi,
\end{aligned}
$$

for any $i, j \in\{1,2\}$. Multiplying this identity by $\left|\partial_{i j}^{2} \mu\right|^{p-2} \partial_{i j}^{2} \mu$, for $p>2$, we find by elementary Sobolev inequalities,

$$
\left\|\partial_{i j}^{2} \mu\right\|_{L^{p}}^{p} \leq C(R, J)\left(\left\|\partial_{i j}^{2} \varphi\right\|_{L^{p}}+\|\varphi\|_{H^{2}}+\|\varphi\|_{H^{2}}^{2}\right)\left\|\partial_{i j}^{2} \mu\right\|_{L^{p}}^{p-1}
$$

owing to the fact that $\varphi \in L^{\infty}\left(0, T ; H^{2}(\Omega)\right)$ and that $J$ is admissible in the sense of Definition 2.4. Therefore,

$$
\left\|\partial_{i j}^{2} \mu(0)\right\|_{L^{p}} \leq C\left(\left\|\varphi_{0}\right\|_{H^{2}}, J,\left\|\partial_{i j}^{2} \varphi(0)\right\|_{L^{p}}\right)
$$

and so $\mu(0) \in W^{2, p}(\Omega)$ turns out to be equivalent to having $\varphi_{0}=\varphi(0) \in W^{2, p}(\Omega)$, for $p \in(2, \infty)$. Now that we have gotten the preliminaries out of the way, we can state and prove the following.

Lemma 3.6. Let $\varphi_{0} \in W^{2, p}(\Omega)$ and $\omega_{0}=\nabla \times u_{0} \in L^{\infty}(\Omega), u \in W^{1,2}(\Omega) \cap H$ for some $p \in(2, \infty)$. Assume that $J$ is admissible and $F \in C^{3}(\mathbb{R}, \mathbb{R})$ obeys (H2). Then the conclusion of Corollary 3.4 is verified. In particular, the solution $(u, \varphi)$ satisfies

$$
\nabla \times u \in L^{\infty}\left(0, T ; L^{\infty}(\Omega)\right), \varphi \in L^{\infty}\left(0, T ; W^{2, p}(\Omega)\right) .
$$

Proof. We first differentiate both equations of (1.1) with respect to time. We have

$$
\partial_{t}^{2} \varphi+\operatorname{div}\left(\partial_{t} u \varphi\right)+u \cdot \nabla \partial_{t} \varphi=-B_{N} \partial_{t} \mu, \text { in } \Omega \times(0, T)
$$

and

$$
\partial_{t} \mu=b(x, \varphi) \partial_{t} \varphi-J * \partial_{t} \varphi, \text { in } \Omega \times(0, T) .
$$

Testing equation (3.42) in $L^{2}(\Omega)$ by $\left|\partial_{t} \varphi\right|^{\lambda-1} \partial_{t} \varphi, \lambda>1$ and using the fact that $u(t), \partial_{t} u(t) \in H$, a.e. $t \in(0, T)$, we derive

$$
\begin{aligned}
& \frac{1}{\lambda+1} \frac{d}{d t}\left\|\partial_{t} \varphi\right\|_{L^{\lambda+1}}^{\lambda+1}+\lambda \int_{\Omega}\left|\partial_{t} \varphi\right|^{\lambda-1} \nabla \partial_{t} \varphi \cdot \nabla \partial_{t} \mu d x \\
& =\lambda \int_{\Omega} \varphi\left|\partial_{t} \varphi\right|^{\lambda-1} \nabla \partial_{t} \varphi \cdot \partial_{t} u d x
\end{aligned}
$$

Applying the gradient on the identity (3.43) and replacing the resulting relation into the second term on the left-hand side, we infer

$$
\frac{d}{d t}\left\|\partial_{t} \varphi\right\|_{L^{\lambda+1}}^{\lambda+1}+\lambda(\lambda+1) c_{0} \int_{\Omega}\left|\partial_{t} \varphi\right|^{\lambda-1}\left|\nabla \partial_{t} \varphi\right|^{2} d x \leq I_{1}+I_{2}+I_{3}
$$

owing to assumption (H2), which states that $b(x, \varphi)=a(x)+F^{\prime \prime}(\varphi) \geq c_{0}$; here, we have set

$$
\left\{\begin{array}{l}
I_{1}:=\lambda(\lambda+1) \int_{\Omega} \varphi\left|\partial_{t} \varphi\right|^{\lambda-1} \nabla \partial_{t} \varphi \cdot \partial_{t} u d x \\
I_{2}:=\lambda(\lambda+1) \int_{\Omega}\left|\partial_{t} \varphi\right|^{\lambda-1} \nabla \partial_{t} \varphi \cdot \nabla J * \partial_{t} \varphi d x \\
I_{3}:=-\lambda(\lambda+1) \int_{\Omega} \partial_{t} \varphi\left|\partial_{t} \varphi\right|^{\lambda-1} \nabla \partial_{t} \varphi \cdot\left(\nabla a+F^{\prime \prime \prime}(\varphi) \nabla \varphi\right) d x
\end{array}\right.
$$


We also note that

$$
\left|\partial_{t} \varphi\right|^{\lambda-1}\left|\nabla \partial_{t} \varphi\right|^{2}=\left.\left.\left(\frac{2}{\lambda+1}\right)^{2}|\nabla| \partial_{t} \varphi\right|^{\frac{\lambda+1}{2}}\right|^{2}
$$

The simplest term $I_{2}$ can be estimated, for any $\epsilon>0$,

$$
\begin{aligned}
\left|I_{2}\right| & \leq \epsilon \lambda(\lambda+1) \int_{\Omega}\left|\partial_{t} \varphi\right|^{\lambda-1}\left|\nabla \partial_{t} \varphi\right|^{2} d x \\
& +C_{\epsilon} \lambda(\lambda+1) \int_{\Omega}\left|\partial_{t} \varphi\right|^{\lambda-1}\left|\nabla J * \partial_{t} \varphi\right|^{2} d x \\
& \leq \epsilon \lambda(\lambda+1) \int_{\Omega}\left|\partial_{t} \varphi\right|^{\lambda-1}\left|\nabla \partial_{t} \varphi\right|^{2} d x \\
& +C_{\epsilon} \lambda(\lambda+1)\left\|\partial_{t} \varphi\right\|_{L^{\lambda+1}}^{\lambda-1}\left(\|\nabla J\|_{L^{1}}\left\|\partial_{t} \varphi\right\|_{L^{\lambda+1}}\right)^{2} \\
& \leq \epsilon \lambda(\lambda+1) \int_{\Omega}\left|\partial_{t} \varphi\right|^{\lambda-1}\left|\nabla \partial_{t} \varphi\right|^{2} d x \\
& +C\left(\epsilon,\|J\|_{W^{1,1}}\right) \lambda(\lambda+1)\left\|\partial_{t} \varphi\right\|_{L^{\lambda+1}}^{\lambda+1} .
\end{aligned}
$$

Concerning $I_{3}$, we have

$$
\begin{aligned}
\left|I_{3}\right| & \leq \epsilon \lambda(\lambda+1) \int_{\Omega}\left|\partial_{t} \varphi\right|^{\lambda-1}\left|\nabla \partial_{t} \varphi\right|^{2} d x \\
& +C\left(\epsilon, R,\|\nabla a\|_{L^{\infty}}\right) \lambda(\lambda+1)\left(\left\|\partial_{t} \varphi\right\|_{L^{\lambda+1}}^{\lambda+1}+\int_{\Omega}\left|\partial_{t} \varphi\right|^{\lambda+1}|\nabla \varphi|^{2} d x\right) .
\end{aligned}
$$

owing to $\|\varphi\|_{L^{\infty}\left(0, T ; L^{\infty}(\Omega)\right)} \leq R$ and the fact that $\nabla a \in L^{\infty}(\Omega)$ (which is satisfied by assumption (H1)). To estimate the last term on the right-hand side of (3.47) we must proceed in a more accurate way. We shall make use of 2D Poincare-Young type inequality which can be proven by a contradiction argument or alternatively, it can be shown as a consequence of the 2D Gagliardo-Nirenbeg-Sobolev inequality. For any $\widetilde{\epsilon}>0$, there exists $0<C=C(\widetilde{\epsilon}, q, r) \sim(\widetilde{\epsilon})^{-\eta}$ (for some $\eta=\eta(q, r)>0$ ) such that

$$
\|\psi\|_{L^{q}}^{2} \leq \widetilde{\epsilon}\|\nabla \psi\|_{L^{2}}^{2}+C\|\psi\|_{L^{r}}^{2}, \text { for } r \in(0, q),
$$

for any $q \in(1, \infty)$. The Holder inequality with exponents $(1+\delta, 1+1 / \delta)$ for any $\delta>0$ yields

$$
\begin{aligned}
\int_{\Omega}\left|\partial_{t} \varphi\right|^{\lambda+1}|\nabla \varphi|^{2} d x & \leq\left(\int_{\Omega}\left|\partial_{t} \varphi\right|^{\frac{\lambda+1}{2} 2(1+\delta)} d x\right)^{1 /(1+\delta)}\left(\int_{\Omega}|\nabla \varphi|^{2(1+1 / \delta)} d x\right)^{\delta /(\delta+1)} \\
& \leq\left\|\partial_{t} \varphi\right\|_{L^{(\lambda+1)(1+\delta)}}^{\lambda+1}\|\nabla \varphi\|_{L^{2(1+1 / \delta)}}^{2} \\
& \leq C(T, R, J)\left\|\partial_{t} \varphi\right\|_{L^{(\lambda+1)(1+\delta)}}^{\lambda+1}
\end{aligned}
$$

owing to the fact that $\varphi \in L^{\infty}\left(0, T ; W^{2,2}(\Omega)\right) \subset L^{\infty}\left(0, T ; W^{1,2(1+1 / \delta)}(\Omega)\right)$, for any $\delta>0$. Further taking $\psi=\left|\partial_{t} \varphi\right|^{\frac{\lambda+1}{2}}$ in the inequality (3.48) with $r=1$, we obtain

$$
\begin{aligned}
\left\|\partial_{t} \varphi\right\|_{L^{(\lambda+1)(1+\delta)}}^{\lambda+1} & =\left(\int_{\Omega}|\psi|^{2(1+\delta)} d x\right)^{1 /(1+\delta)}=\|\psi\|_{L^{2(1+\delta)}}^{2} \\
& \leq \widetilde{\epsilon}\left\|\nabla\left|\partial_{t} \varphi\right|^{\frac{\lambda+1}{2}}\right\|_{L^{2}}^{2}+C(\widetilde{\epsilon}, \delta)\left\|\left|\partial_{t} \varphi\right|^{\frac{\lambda+1}{2}}\right\|_{L^{1}}^{2}
\end{aligned}
$$




$$
=\frac{4 \widetilde{\epsilon}}{(\lambda+1)^{2}} \int_{\Omega}\left|\partial_{t} \varphi\right|^{\lambda-1}\left|\nabla \partial_{t} \varphi\right|^{2} d x+C(\widetilde{\epsilon}, \delta)\left\|\partial_{t} \varphi\right\|_{L^{\lambda+1}}^{\lambda+1}
$$

by recalling (3.45). Combining (3.50) together with (3.49), we then infer from (3.47) that

$$
\left|I_{3}\right| \leq 2 \epsilon \lambda(\lambda+1) \int_{\Omega}\left|\partial_{t} \varphi\right|^{\lambda-1}\left|\nabla \partial_{t} \varphi\right|^{2} d x+C(\lambda, T, R, J)\left\|\partial_{t} \varphi\right\|_{L^{\lambda+1}}^{\lambda+1} .
$$

by choosing $\widetilde{\epsilon}<\widetilde{e}_{0}(T, \epsilon, R, J, \lambda)$ appropriately small, depending on $C(T, R, J)$ and $\lambda>1$. The dependance of the constant $C>0$ on the right-hand side of (3.51) in $\lambda>0$ can be made explicit (i.e., $C(\lambda, \cdot, \cdot, \cdot) \sim \lambda^{\gamma}$, for some $\gamma>0$ independent of $T, \lambda, R)$. The final term $I_{1}$ can be estimated in a similar way. For any $\epsilon>0$, we have

$$
\begin{aligned}
\left|I_{1}\right| & \leq \epsilon \lambda(\lambda+1) \int_{\Omega}\left|\partial_{t} \varphi\right|^{\lambda-1}\left|\nabla \partial_{t} \varphi\right|^{2} d x+C(\epsilon, R) \lambda(\lambda+1) \int_{\Omega}\left|\partial_{t} \varphi\right|^{\lambda-1}\left|\partial_{t} u\right|^{2} d x \\
& \leq 2 \epsilon \lambda(\lambda+1) \int_{\Omega}\left|\partial_{t} \varphi\right|^{\lambda-1}\left|\nabla \partial_{t} \varphi\right|^{2} d x+C(\lambda, T, R, J)\left\|\partial_{t} \varphi\right\|_{L^{\lambda+1}}^{\lambda+1} .
\end{aligned}
$$

where the last term on right-hand side of the first inequality (3.52) can be estimated as in (3.49)-(3.51). Indeed, recall that since $\omega_{0} \in L^{\infty}(\Omega) \subset L^{q}(\Omega)$, there holds $\omega \in L^{\infty}\left(0, T ; L^{q}(\Omega)\right)$, for any $q \in[2, \infty)$ and $\partial_{t} u \in L^{\infty}\left(0, T ; L^{q}(\Omega)\right)$ as well, owing to the conclusion of Lemma 3.5 (see (3.35)). Putting all the estimates (3.46), (3.51) and (3.52) together in (3.44) and choosing a sufficiently small $\epsilon \leq c_{0} / 10$, we arrive at the inequality

$$
\frac{d}{d t}\left\|\partial_{t} \varphi\right\|_{L^{\lambda+1}}^{\lambda+1}+\left.\left.\frac{c_{0} \lambda}{\lambda+1} \int_{\Omega}|\nabla| \partial_{t} \varphi\right|^{\frac{\lambda+1}{2}}\right|^{2} d x \leq C(\lambda, R, T, J)\left\|\partial_{t} \varphi\right\|_{L^{\lambda+1}}^{\lambda+1}
$$

Integrating now (3.53) in time, we immediately deduce that

$$
\partial_{t} \varphi \in L^{\infty}\left(0, T ; L^{\lambda+1}(\Omega)\right),
$$

for any $\lambda>1$ for as long as $\partial_{t} \varphi(0) \in L^{\lambda+1}(\Omega)$. In particular, it follows that (3.38) holds with $p=\lambda+1$ and so a simple argument like in (3.40) involving the identity (3.39) gives $\varphi \in L^{\infty}\left(0, T ; W^{2, \lambda+1}(\Omega)\right)$, which is the desired claim in (3.41). The first of (3.41) is already a consequence of this estimate and the statement of Corollary 3.4. The proof of Lemma 3.6 is thus concluded.

Theorem 3.7. Let the assumptions of Lemma 3.6 be satisfied and assume in addition that $u_{0} \in W^{3,2}(\Omega) \cap H$. Then the solution $(u, \varphi)$ also has the following regularity

$$
u \in L^{\infty}\left(0, T ; W^{3,2}(\Omega) \cap H\right) .
$$

Proof. We apply the gradient $\nabla$ to the vorticity equation. We have

$$
\partial_{t}(\nabla \omega)=-\nabla(u \cdot \nabla \omega)-\nabla\left(\nabla \mu \cdot \nabla^{\perp} \varphi\right) \text { in } \Omega \times(0, T) .
$$

Testing this equation in $L^{2}(\Omega)$ by $\nabla \omega$ we deduce

$$
\begin{aligned}
\frac{1}{2} \frac{d}{d t}\|\nabla \omega\|_{L^{2}}^{2} & =-\int_{\Omega} \nabla(u \cdot \nabla \omega) \cdot \nabla \omega d x-\int_{\Omega} \nabla\left(\nabla \mu \cdot \nabla^{\perp} \varphi\right) \cdot \nabla \omega d x \\
& =K_{1}+K_{2} .
\end{aligned}
$$


We first have using the usual Einstein summation convention, and the fact that $u$ is divergent free and $u \cdot n=0$ on $\partial \Omega$,

$$
\begin{aligned}
K_{1} & =-\int_{\Omega} \partial_{m}\left(u_{k} \partial_{k} \omega\right) \partial_{m} \omega d x \\
& =-\int_{\Omega} \partial_{m} u_{k} \partial_{k} \omega \partial_{m} \omega d x-\frac{1}{2} \int_{\Omega} u_{k} \partial_{k}\left(\partial_{m} \omega\right)^{2} d x \\
& =-\int_{\Omega} \partial_{m} u_{k} \partial_{k} \omega \partial_{m} \omega d x \\
& \leq\|\nabla u\|_{L^{\infty}}\|\nabla \omega\|_{L^{2}}^{2} .
\end{aligned}
$$

At this point we shall apply the following inequality (cf., e.g., [7, Lemma 4.3]) with $a>0$,

$$
\|v\|_{L^{\infty}} \leq C \sup _{q \geq 2} \frac{\|v\|_{L^{q}}}{q^{a}}\left(\ln \left(e+\|v\|_{H^{2}}\right)\right)^{a},
$$

for some constant $C>0$ independent of $v$. We note that the inequality in $[7$, Lemma 4.3] was stated in the case $\Omega=\mathbb{R}^{2}$ but it also holds in any bounded domain $\Omega \subset \mathbb{R}^{2}$ with a boundary of class $C^{3}$ by simply exploiting a suitable extension operator $E: W^{2,2}(\Omega) \rightarrow W^{2,2}\left(\mathbb{R}^{2}\right)$, given by $E v=\widetilde{v}$ with the extension $\widetilde{v} \in C_{b}\left(\mathbb{R}^{2}\right)$ satisfying (3.57). Appealing also to the fact that $\|\nabla u\|_{W^{2,2}} \leq C\|\omega\|_{W^{1,2}}$ owing to [3, Lemma 5] and $\|\omega\|_{L^{\infty}\left(0, T ; L^{\infty}(\Omega)\right)} \leq C_{T}$, then from (3.56) we obtain

$$
\begin{aligned}
\left|K_{1}\right| & \leq C \sup _{q \geq 2} \frac{\|\nabla u\|_{L^{q}}}{q}\left(\ln \left(e+\|\omega\|_{W^{1,2}}\right)\right) \\
& \leq C \sup _{q \geq 2}\|\omega\|_{L^{q}}\left(\ln \left(e+\|\omega\|_{W^{1,2}}\right)\right) \\
& \leq C(T, R, J, \Omega)\left(\ln \left(e+\|\omega\|_{W^{1,2}}\right)\right),
\end{aligned}
$$

by means of the Biot-Savart inequality (2.2). On the other hand, for some $p>2$, $K_{2}$ can be estimated as follows:

$$
\begin{aligned}
K_{2} & =-\int_{\Omega} \partial_{m}\left(\partial_{k} \mu \partial_{k}^{\perp} \varphi\right) \partial_{m} \omega d x \\
& =-\int_{\Omega} \partial_{m k}^{2} \mu \partial_{k}^{\perp} \varphi \partial_{m} \omega d x-\int_{\Omega} \partial_{k} \mu\left(\partial_{m} \partial_{k}^{\perp} \varphi\right) \partial_{m} \omega d x \\
& \lesssim\|\nabla \omega\|_{L^{2}}\left(\left\|\nabla^{\perp} \varphi\right\|_{L^{\infty}}\|\mu\|_{H^{2}}+\|\nabla \mu\|_{L^{\infty}}\|\varphi\|_{H^{2}}\right) \\
& \leq C(T, R, J)\|\nabla \omega\|_{L^{2}}\left(\|\varphi\|_{W^{2, p}}+\|\mu\|_{W^{2, p}}\right) \\
& \leq C\left(1+\|\nabla \omega\|_{L^{2}}^{2}\right)
\end{aligned}
$$

Set now $z(t):=\|\omega(t)\|_{W^{1,2}}^{2}$. Inserting the estimates (3.58)-(3.59) into the righthand side of (3.46) and adding the resulting inequality to that of (3.29) with $q=2$, we obtain

$$
\frac{d}{d t} z(t) \leq C(T, R, \Omega, J) \ln (e+z(t)) z(t), t \in(0, T) .
$$

Integrating this inequality over time $t \in(0, T)$, we deduce

$$
\|\omega(t)\|_{W^{1,2}}^{2} \leq C(T, R, J, \Omega) \sim e^{e^{C T}}, t \in(0, T) .
$$

Thus since $\|\nabla u\|_{W^{2,2}} \leq C\|\omega\|_{W^{1,2}}$, (3.60) yields the corresponding regularity in (3.54). This completes the proof. 
We shall now focus our attention to showing the uniqueness of solutions in the class of bounded vorticities. Although this is just some variation of the famous Yudovich theorem (see [29]), for the convenience of the reader, we give below an explicit proof of this result for our inviscid system (1.1)-(1.5). Theorem 2.5 follows then from Theorem 3.8 below.

Theorem 3.8. Let $\left(u_{i}, \varphi_{i}\right)$ be any two solutions, which are at least regular as in Definition 2.1, such that the corresponding vorticities

$$
\omega_{i}=\nabla \times u_{i} \in L^{\infty}\left(0, T ; L^{\infty}(\Omega)\right) .
$$

Then $u_{1} \equiv u_{2}, \varphi_{1} \equiv \varphi_{2}$ in $\Omega \times(0, T)$ provided that $\left(u_{1}(0), \varphi_{1}(0)\right) \equiv\left(u_{2}(0), \varphi_{2}(0)\right)$.

Proof. Let us set $u(t)=u_{1}(t)-u_{2}(t)$ and $\varphi(t)=\varphi_{1}(t)-\varphi_{2}(t)$. Consider

$$
Y(t):=\|u(t)\|_{L^{2}}^{2}+\left\|B_{N}^{-1 / 2}(\varphi(t)-\bar{\varphi}(t))\right\|_{L^{2}}^{2}
$$

and observe that by Definition 2.1 and (3.61), $(u, \varphi) \in C\left([0, T] ; H \times L^{2}(\Omega)\right)$ and $Y$ is absolutely continuous on $(0, T)$. First, exactly as in [11] rewriting the Korteweg force as

$$
K(\varphi)=-\nabla a \frac{\varphi^{2}}{2}-(J * \varphi) \nabla \varphi
$$

by incorporating any potential terms in the pressure $\tilde{\pi}:=\pi-F(\varphi)+a \varphi^{2} / 2$, we see that the difference $(u(t), \varphi(t))$ satisfies the system

$$
\partial_{t} \varphi=-B_{N} \widetilde{\mu}-u \cdot \nabla \varphi_{1}-u_{2} \cdot \nabla \varphi, \widetilde{\mu}=a \varphi-J * \varphi+F^{\prime}\left(\varphi_{2}\right)-F^{\prime}\left(\varphi_{1}\right),
$$

$$
\partial_{t} u+\left(u_{1} \cdot \nabla\right) u+(u \cdot \nabla) u_{2}+\nabla \widetilde{\pi}=-\varphi\left(\varphi_{1}+\varphi_{2}\right) \frac{\nabla a}{2}-(J * \varphi) \nabla \varphi_{2}-\left(J * \varphi_{1}\right) \nabla \varphi,
$$

where $\tilde{\pi}:=\widetilde{\pi}_{2}-\widetilde{\pi}_{1}$ and $\operatorname{div}(u)=0, u \cdot n=0$ on $\partial \Omega$. Moreover, by (3.61) and (2.2) we know that $u_{i} \in L^{\infty}\left(0, T ; W^{1, p}(\Omega)\right)$ for any $p \in(2, \infty)$, and so

$$
\left\|\nabla u_{i}(t)\right\|_{L^{p}} \leq C p, t \in[0, T] .
$$

We multiply (3.64) by $u$ in $H$ and the first of (3.63) by $B_{N}^{-1}(\varphi-\langle\varphi\rangle$ ) (notice that we also have $\langle\varphi\rangle=\left\langle\varphi_{1}(0)\right\rangle-\left\langle\varphi_{2}(0)\right\rangle \equiv 0$ since $\varphi_{1}(0) \equiv \varphi_{2}(0)$ by assumption). After standard transformations, we arrive at

$$
\frac{d}{d t} Y(t)+2\left(a(x) \varphi+F^{\prime}\left(\varphi_{1}\right)-F^{\prime}\left(\varphi_{2}\right), \varphi\right) \leq 2 \sum_{j=1}^{6}\left|I_{j}\right|,
$$

where

$$
\left\{\begin{array}{l}
I_{1}=-\frac{1}{2}\left(\varphi\left(\varphi_{1}+\varphi_{2}\right) \nabla a, u\right), I_{2}=-\left((J * \varphi) \nabla \varphi_{2}, u\right) \\
I_{3}=-\left(\left(J * \varphi_{1}\right) \nabla \varphi, u\right), I_{4}=-\left(u \cdot \nabla \varphi_{1}, B_{N}^{-1}(\varphi-\langle\varphi\rangle)\right) \\
I_{5}=-\left(u_{2} \cdot \nabla \varphi, B_{N}^{-1}(\varphi-\langle\varphi\rangle)\right), I_{6}=-\left(u \cdot \nabla u_{2}, u\right) .
\end{array}\right.
$$

Since $\varphi_{i} \in L^{\infty}\left(0, T ; L^{\infty}(\Omega)\right)$ and $J \in W_{\text {loc }}^{1,1}$, we estimate $I_{1}-I_{3}$ in the following simple way:

$$
\begin{aligned}
& \left|I_{1}\right| \leq\|\varphi\|_{L^{2}}\left\|\varphi_{1}+\varphi_{2}\right\|_{L^{\infty}}\|\nabla a\|_{L^{\infty}}\|u\|_{L^{2}} \\
& \leq \epsilon\|\varphi\|_{L^{2}}^{2}+C(\epsilon, R, J)\|u\|_{L^{2}}^{2} \\
& \left|I_{2}\right|=\left|\left(\varphi_{2},(\nabla J * \varphi) u\right)\right| \leq\left\|\varphi_{2}\right\|_{L^{\infty}}\|\nabla J * \varphi\|_{L^{2}}\|u\|_{L^{2}}
\end{aligned}
$$




$$
\begin{aligned}
& \leq \epsilon\|\varphi\|_{L^{2}}^{2}+C(\epsilon, R, J)\|u\|_{L^{2}}^{2}, \\
& \left|I_{3}\right|=\left|\left(\left(\nabla J * \varphi_{1}\right) \varphi, u\right)\right| \leq\left\|\nabla J * \varphi_{1}\right\|_{L^{\infty}}\|\varphi\|\|u\|_{L^{2}} \\
& \leq \epsilon\|\varphi\|_{L^{2}}^{2}+C(\epsilon, R, J)\|u\|_{L^{2}}^{2},
\end{aligned}
$$

for any $\epsilon>0$. Since $u_{i} \in L^{\infty}\left(0, T ; L^{\infty}(\Omega)\right)$ by (3.65) we also have

$$
\begin{aligned}
& \left|I_{4}\right|=\left|\left(u \cdot \nabla B_{N}^{-1}(\varphi-\langle\varphi\rangle), \varphi_{1}\right)\right| \leq\|u\|_{L^{2}}\left\|\nabla B_{N}^{-1}(\varphi-\langle\varphi\rangle)\right\|_{L^{2}}\left\|\varphi_{1}\right\|_{L^{\infty}} \\
& \leq\|u\|_{L^{2}}^{2}+C(R)\left\|B_{N}^{-1 / 2}(\varphi-\langle\varphi\rangle)\right\|_{L^{2}}^{2}, \\
& \left|I_{5}\right|=\left|\left(u_{2} \cdot \nabla B_{N}^{-1}(\varphi-\langle\varphi\rangle), \varphi\right)\right| \leq\|\varphi\|_{L^{2}}\left\|u_{2}\right\|_{L^{\infty}}\left\|\nabla B_{N}^{-1}(\varphi-\langle\varphi\rangle)\right\|_{L^{2}} \\
& \leq \epsilon\|\varphi\|_{L^{2}}^{2}+C(R, J)\left\|\nabla B_{N}^{-1}(\varphi-\langle\varphi\rangle)\right\|_{L^{4}}^{2} .
\end{aligned}
$$

For $p>1$ arbitrary and $1 / p+1 / p_{*}=1$, by virtue of the interpolation inequality $\|u\|_{L^{2 p_{*}}} \leq\|u\|_{L^{2}}^{1-1 / p}\|u\|_{L^{\infty}}^{1 / p}$, the final term is estimated as follows:

$$
\begin{aligned}
\left|I_{6}\right| & =\left|\left(u \cdot \nabla u_{2}, u\right)\right| \leq C\|u\|_{L^{2 p_{*}}}^{2}\left\|\nabla u_{2}\right\|_{L^{p}} \\
& \leq C p\|u\|_{L^{2}}^{2(1-1 / p)} \leq C p Y(t)^{1-1 / p} .
\end{aligned}
$$

Collecting all the estimates from (3.67)-(3.72) into (3.66), then exploiting assumption (H2) (which yields $\left.\left(a(x) \varphi+F^{\prime}\left(\varphi_{1}\right)-F^{\prime}\left(\varphi_{2}\right), \varphi\right) \geq c_{0}\|\varphi\|_{L^{2}}^{2}\right)$ and choosing a sufficiently small $\epsilon \leq c_{0} / 4$, we obtain

$$
\frac{d}{d t} Y(t) \leq C p Y(t)^{1-1 / p}+C Y(t), \quad t \in(0, T)
$$

for any $p>1$. Since we need an estimate for $Y(t) \leq 1$ small only (recall that $Y(t)$ remains always bounded on $[0, T])$, from $(3.73)$ we infer

$$
\frac{d}{d t} Y(t) \leq C p Y(t)^{1-1 / p}
$$

for some constant $C=C(R, J)>0$ independent of $p$. Take $p=\log (K / E(t))$ for some sufficiently large $K>0$ such that $p>1$. Therefore, we deduce

$$
\frac{d}{d t} Y(t) \leq C Y(t) \log \frac{K}{Y(t)},
$$

which can be integrated in time over the interval $(\delta, t)$, for some $t>\delta$ to find

$$
Y(t) \leq K\left(\frac{Y(\delta)}{K}\right)^{e^{-C(t-\delta)}}
$$

Passing to the limit as $\delta \rightarrow 0^{+}$into the foregoing inequality and recalling that $Y \in C[0, T]$, we derive that $Y(t)=0$ on $[0, T]$. The proof of Theorem 3.8 is concluded.

Remark 3.2. We briefly explain how the case $\Omega=\mathbb{R}^{2}$ (or $\Omega \subset \mathbb{R}^{2}$ is a smooth compact manifold without boundary) can be handled with our present analysis. We shall focus mainly on the case $\Omega=\mathbb{R}^{2}$ since the case of a compact manifold without boundary can be actually reduced to this. The energy estimate provided by the statement of Proposition 3.1 holds without any modifications also in the case $\Omega=\mathbb{R}^{2}$ (refer also [7] to in the classical case). Relying on the fact that the nonlocal Cahn-Hilliard equation is a parabolic equation of second-order, the bound $\varphi \in L^{\infty}\left(0, T ; L^{\infty}\left(\mathbb{R}^{2}\right)\right)$ is obtained exactly in the same fashion as in [4]. The energy estimate (3.13) produced by the statement of Theorem 3.2 can still be recovered since both the Trudinger and the Biot-Savart inequalities (2.1), (2.2) are 
still valid in $\mathbb{R}^{2}$. The Holder regularity result in [11, Lemma 2] requires no essential modifications while the other remaining statements of Lemmas 3.3, 3.5, 3.6 and Theorem 3.7 can be easily reproduced by energy methods.

We now conclude that both Theorem 2.6 and Theorem 2.3 follow from the statements of the results proven in this section, and a proper approximation scheme for problem (1.1)-(1.5) that we explain in the sequel. Let $\varepsilon \in(0,1)$ be a given small parameter and for some $R>0$, let

$$
\bar{\varphi} \in Z=\left\{\bar{\varphi} \in L^{\infty}\left(0, T ; L^{\infty}(\Omega)\right) \cap L^{2}\left(0, T ; W^{1,2}(\Omega)\right):\|\bar{\varphi}\|_{Z} \leq R\right\} .
$$

Also observe that $Z$ is a closed convex subset of $L^{\infty}\left(0, T ; L^{\infty}(\Omega)\right) \cap L^{2}\left(0, T ; W^{1,2}(\Omega)\right)$ when endowed with the corresponding metric topology. Consider next a further regularizing sequence $\bar{\varphi}^{\varepsilon}$ for $\bar{\varphi}$ such that $\bar{\varphi}^{\varepsilon} \in L^{\infty}\left(0, T ; W^{2, p}(\Omega)\right)$ for some $p>2$, such that

$$
\left\|\bar{\varphi}^{\varepsilon}\right\|_{Z} \leq\|\bar{\varphi}\|_{Z}
$$

uniformly with respect to $\varepsilon \in(0,1)$. We rewrite the Kordeweg force $\mu \nabla \varphi$ as $(a(x) \varphi-J * \varphi) \nabla \varphi$ by incorporating the remainder $\nabla F(\varphi)$ into the pressure term. Further, for any given initial data $\varphi_{0} \in W=W^{1,2}(\Omega) \cap L^{\infty}(\Omega), u_{0} \in V=$ $W^{1,2}(\Omega) \cap H$, consider smooth sequences of data $\left\{\varphi_{0, \varepsilon}\right\} \subset W^{2, p}(\Omega),\left\{u_{0, \varepsilon}\right\} \subset$ $W^{3,2}(\Omega) \cap H$ such that the following hold uniformly in $\varepsilon$ :

$$
\left\|\varphi_{0, \varepsilon}\right\|_{W} \leq\left\|\varphi_{0}\right\|_{W} \text { and }\left\|u_{0, \varepsilon}\right\|_{V} \leq\left\|u_{0}\right\|_{V} \text {. }
$$

We can consider the following Euler equation with both a smooth initial datum and smooth forcing as follows:

$$
\left\{\begin{array}{l}
\partial_{t} u+(u \cdot \nabla) u+\nabla \widetilde{\pi}=\left(a(x) \bar{\varphi}^{\varepsilon}-J * \bar{\varphi}^{\varepsilon}\right) \nabla \bar{\varphi}^{\varepsilon} \\
\operatorname{div}(u)=0, u \cdot n=0 \text { on } \partial \Omega, \\
u_{\mid t=0}=u_{0, \varepsilon} .
\end{array}\right.
$$

Denote the corresponding solution by $u_{\varepsilon}$ and the corresponding pressure by $\widetilde{\pi}_{\varepsilon}$. Let us denote

$$
f_{\varepsilon}=\left(a(x) \bar{\varphi}^{\varepsilon}-J * \bar{\varphi}^{\varepsilon}\right) \nabla \bar{\varphi}^{\varepsilon}
$$

and note that

$$
\operatorname{curl}\left(f_{\varepsilon}\right)=-\left(\nabla a \bar{\varphi}^{\varepsilon}+a \nabla \bar{\varphi}^{\varepsilon}-\nabla J * \bar{\varphi}^{\varepsilon}\right) \cdot \nabla^{\perp} \bar{\varphi}^{\varepsilon} \in L^{\infty}\left(0, T ; L^{\infty}(\Omega)\right)
$$

as well as

$$
\nabla \operatorname{curl}\left(f_{\varepsilon}\right) \in L^{\infty}\left(0, T ; L^{2}(\Omega)\right)
$$

owing to the fact that $\bar{\varphi}^{\varepsilon} \in L^{\infty}\left(0, T ; W^{2, p}(\Omega)\right)$ and $J$ is admissible in the sense of Definition 2.4. Since $u_{0, \varepsilon}$ is also smooth by construction, it is easy to see from the proof of Theorem 3.7 that

$$
u_{\varepsilon} \in L^{\infty}\left(0, T ; W^{3,2}(\Omega) \cap H\right) \cap W^{1, \infty}\left(0, T ; W^{1,2}(\Omega) \cap H\right) .
$$

The second regularity in (3.77) is immediate by virtue of the first of (3.77) and a similar argument that we have employed for (3.36). Next, for the solution $u_{\varepsilon}$ obtained by this procedure we can solve the parabolic problem associated with the nonlocal Cahn-Hilliard equation as follows:

$$
\left\{\begin{array}{l}
\partial_{t} \varphi+u_{\varepsilon} \cdot \nabla \varphi=-B_{N} \mu, \mu=a(x) \varphi-J * \varphi+F^{\prime}(\varphi), \\
\varphi_{\mid t=0}=\varphi_{0, \varepsilon} .
\end{array}\right.
$$


We denote its corresponding solution by $\varphi_{\varepsilon}$ and the chemical potential by $\mu_{\varepsilon}$. We observe that due to the regularity proven for (3.78) in this section (cf. also [11]), we have

$$
\varphi_{\varepsilon} \in C^{\beta, \beta / 2}(\bar{\Omega} \times[0, T]) \cap L^{\infty}\left(0, T ; W^{2, p}(\Omega)\right) \cap W^{1, \infty}\left(0, T ; L^{p}(\Omega)\right)
$$

with some similar properties for $\mu_{\varepsilon}$. Thus, we define a mapping $S_{\varepsilon}(\bar{\varphi})=\varphi_{\varepsilon}$ on which we aim to apply the Schauder fixed point theorem. In order to do so, we must check the following properties:

(1) $S_{\varepsilon}$ is well defined as a mapping from $Z$ into $Z$. In particular, it suffices to check that

$$
\left\|\varphi_{\varepsilon}\right\|_{Z}^{2} \leq R_{0}
$$

for some constant $R_{0}>0$ which is independent of $R>0$ and $\varepsilon \in(0,1)$.

(2) We have $\varphi_{\varepsilon} \in C^{\beta, \beta / 2}(\bar{\Omega} \times[0, T]) \cap L^{2}\left(0, T ; W^{2,2}(\Omega)\right)$, which is in fact already a consequence of (3.79). Note that this property entails that $S_{\varepsilon}$ is a compact mapping.

(3) The mapping $S_{\varepsilon}$ is continuous on $Z$. In particular, owing to the regularity (3.79) it suffices to show that

$$
\left\|\varphi_{1 \varepsilon}-\varphi_{2 \varepsilon}\right\|_{\left(W^{1,2}\right)^{*}} \leq R_{1}\left\|\bar{\varphi}_{1}-\bar{\varphi}_{2}\right\|_{Z}
$$

where $\varphi_{i \varepsilon}$ are any two solutions, satisfying (3.79), such that $S_{\varepsilon}\left(\bar{\varphi}_{i}\right)=\varphi_{i \varepsilon}$, for given $\bar{\varphi}_{i} \in Z_{R}, i=1,2$. Here the constant $R_{1}>0$ may also depend on $\varepsilon \in(0,1)$.

After these conditions are satisfied, we can apply the conclusion of the Schauder fixed point theorem to infer the existence of at least one fixed point $\varphi_{\varepsilon}$ such that $S_{\varepsilon}\left(\varphi_{\varepsilon}^{\varepsilon}\right)=\varphi_{\varepsilon}$. In particular, such a fixed point solution will satisfy (3.79) and therefore the solution $u_{\varepsilon}$ of the Euler equation (3.76) will also be smooth in the class of (3.77). In particular, these considerations allow us to perform rigorously the required computations and estimates in this section. In order to ensure some compactness for these approximating sequence of solutions $\left(u_{\varepsilon}, \varphi_{\varepsilon}\right)$, and properly pass to the limit as $\varepsilon \rightarrow 0$, to deduce the existence results stated in Theorem 2.3 and Theorem 2.6, we would require to obtain uniform estimates with respect to $\varepsilon$. For instance, for the statement of Theorem 2.3, we would need to show:

(4) For any $T>0$, we have

$$
\left\|u_{\varepsilon}\right\|_{L^{\infty}(0, T ; V)} \leq C, \quad\left\|\varphi_{\varepsilon}\right\|_{L^{\infty}(0, T ; W)} \leq C
$$

for some constant $C>0$ independent of $\varepsilon$. Indeed, as these uniform bounds are realized, it can be easily checked that the limit function of $\left(u_{\varepsilon}, \varphi_{\varepsilon}\right)$ as $\varepsilon \rightarrow 0$ is indeed a weak solution of (1.1)-(1.5) in the sense of Definition 2.1. Higher-order estimates can be also performed uniformly with respect to $\varepsilon \in(0,1)$ in light of the proofs of Lemmas 3.3, 3.5, 3.6 and Theorem 3.7. Thus a passage to the limit in these estimates can be performed as well and thus one can also verify the whole statement of Theorem 2.6. As usual, these arguments can be concluded by noticing that $T>0$ is arbitrary in all of these estimates.

Let us now briefly explain how to get all of (1)-(4). To show (1), we first multiply the first equation of (3.76) in $H$ by $u=u_{\varepsilon}$, to deduce

$$
\frac{d}{d t}\left\|u_{\varepsilon}\right\|_{L^{2}}^{2} \leq 2\left\|\left(a(x) \bar{\varphi}^{\varepsilon}-J * \bar{\varphi}^{\varepsilon}\right) \nabla \bar{\varphi}^{\varepsilon}\right\|_{L^{2}}\left\|u_{\varepsilon}\right\|_{L^{2}} .
$$


In particular, owing to (3.75) and (3.74) this estimate yields

$$
\begin{aligned}
\left\|u_{\varepsilon}(t)\right\|_{L^{2}}^{2} & \leq 2\left\|u_{0}\right\|_{L^{2}}^{2}+8\left(\int_{0}^{T}\left\|\left(a(x) \bar{\varphi}^{\varepsilon}-J * \bar{\varphi}^{\varepsilon}\right) \nabla \bar{\varphi}^{\varepsilon}\right\|_{L^{2}} d s\right)^{2} \\
& \leq 2\left\|u_{0}\right\|_{L^{2}}^{2}+C(J)\left(R^{2} T^{2}+R^{3} T\right)
\end{aligned}
$$

for some $C=C(J)>0$ independent of $\varepsilon, R$, for any given $R>0$ and $T>0$. In particular, this shows that $u_{\varepsilon} \in L^{\infty}(0, T ; H)$ uniformly bounded for any $\varepsilon \in(0,1)$. With this information we can proceed to obtain an estimate for the solution $\varphi_{\varepsilon}$ of (3.78) as follows. We multiply the first equation of (3.78) by $\varphi$ and integrate by parts over $\Omega$. We derive

$$
\begin{aligned}
& \frac{d}{d t}\left\|\varphi_{\varepsilon}\right\|_{L^{2}}^{2}+2 \int_{\Omega}\left(a(x)+F^{\prime \prime}\left(\varphi_{\varepsilon}\right)\right)\left|\nabla \varphi_{\varepsilon}\right|^{2} d x \\
& =2\left(\nabla J * \varphi_{\varepsilon}, \nabla \varphi_{\varepsilon}\right)-2\left(\nabla a \varphi_{\varepsilon}, \nabla \varphi_{\varepsilon}\right) \\
& \leq C\left(\epsilon,\|J\|_{W^{1,1}}\right)\left\|\varphi_{\varepsilon}\right\|_{L^{2}}^{2}+2 \epsilon\left\|\nabla \varphi_{\varepsilon}\right\|_{L^{2}}^{2},
\end{aligned}
$$

for any $\epsilon>0$. By assumption (H2) we can then absorb the small $\epsilon$-term on the left hand side. Integrating the resulting inequality over time and recalling (3.75), we derive

$$
\left\|\varphi_{\varepsilon}\right\|_{L^{\infty}\left(0, T ; L^{2}(\Omega)\right) \cap L^{2}\left(0, T ; W^{1,2}(\Omega)\right)} \leq C\left(T,\left\|\varphi_{0}\right\|_{L^{2}}\right),
$$

where the constant on the right-hand side is clearly independent of $\varepsilon>0$. Since $u_{\varepsilon} \in L^{\infty}(0, T ; H)$ is divergent free and $u_{\varepsilon} \cdot n=0$ on $\partial \Omega$, we can also multiply the first of (3.78) by $\left|\varphi_{\varepsilon}\right|^{p-1} \varphi_{\varepsilon}$ and perform a Moser-like iteration as in the proof of [4, Theorem 2.1] to find that

$$
\left\|\varphi_{\varepsilon}\right\|_{L^{\infty}\left(0, T ; L^{\infty}(\Omega)\right)} \leq C\left(\left\|\varphi_{0, \varepsilon}\right\|_{L^{\infty}(\Omega)},\left\|\varphi_{\varepsilon}\right\|_{L^{\infty}\left(0, T ; L^{2}(\Omega)\right)}\right) .
$$

Then owing to (3.83) and (3.75) we also arrive at $\varphi_{\varepsilon} \in L^{\infty}\left(0, T ; L^{\infty}(\Omega)\right)$ uniformly with a constant $R_{0}=R_{0}\left(T,\left\|\varphi_{0}\right\|_{L^{\infty}(\Omega)}\right)$ that is independent in $\varepsilon>0$ and $R>0$; together with (3.83) it concludes the proof of property (1). In particular, we also have

$$
\mu_{\varepsilon} \in L^{2}\left(0, T ; W^{1,2}(\Omega)\right) \cap L^{\infty}\left(0, T ; L^{\infty}(\Omega)\right)
$$

uniformly with respect to $\varepsilon \in(0,1)$. Note that the proceeding uniform bounds were the starting point of the proof of Theorem 3.2. Hence, it can be concluded that the approximate solutions $\left(u_{\varepsilon}, \varphi_{\varepsilon}\right)$ satisfy (3.9)-(3.12) there uniformly with respect to $\varepsilon$. In particular, (3.80) is indeed verifiable. It follows that the second property (2) is also satisfied. To show the continuity of the mapping $S_{\varepsilon}$, we consider the differences $\bar{\varphi}=\bar{\varphi}_{1}-\bar{\varphi}_{2}, u_{\varepsilon}=u_{1 \varepsilon}-u_{2 \varepsilon}, \varphi_{\varepsilon}=\varphi_{1 \varepsilon}-\varphi_{2 \varepsilon}$, and observe that $\left(u_{\varepsilon}, \varphi_{\varepsilon}\right)$ satisfies the following problem

$$
\begin{gathered}
\partial_{t} \varphi_{\varepsilon}=-B_{N} \widetilde{\mu}_{\varepsilon}-u_{\varepsilon} \cdot \nabla \varphi_{1 \varepsilon}-u_{2 \varepsilon} \cdot \nabla \varphi_{\varepsilon}, \\
\widetilde{\mu}_{\varepsilon}=a \varphi_{\varepsilon}-J * \varphi_{\varepsilon}+F^{\prime}\left(\varphi_{2 \varepsilon}\right)-F^{\prime}\left(\varphi_{1 \varepsilon}\right), \\
\partial_{t} u_{\varepsilon}+\left(u_{1 \varepsilon} \cdot \nabla\right) u_{\varepsilon}+\left(u_{\varepsilon} \cdot \nabla\right) u_{2 \varepsilon}+\nabla \widetilde{\pi}_{\varepsilon}=\bar{g}^{\varepsilon},
\end{gathered}
$$

with $u_{\varepsilon}(0) \equiv 0, \varphi_{\varepsilon}(0) \equiv 0$. Here we have set

$$
\bar{g}^{\varepsilon}=\left(a(x) \bar{\varphi}^{\varepsilon}-J * \bar{\varphi}^{\varepsilon}\right) \nabla \bar{\varphi}_{1}^{\varepsilon}+\left(a(x) \bar{\varphi}_{2}^{\varepsilon}-J * \bar{\varphi}_{2}^{\varepsilon}\right) \nabla \bar{\varphi}^{\varepsilon} .
$$


Testing first equation (3.87) in $H$ by $u_{\varepsilon}$, by standard estimates we deduce

$$
\begin{aligned}
\frac{d}{d t}\left\|u_{\varepsilon}\right\|_{L^{2}}^{2} & \leq 2\left\|u_{\varepsilon}\right\|_{L^{2}}^{2}\left\|\nabla u_{2 \varepsilon}\right\|_{L^{\infty}}+\left\|\bar{g}^{\varepsilon}\right\|_{L^{2}}\left\|u_{\varepsilon}\right\|_{L^{2}} \\
& \lesssim\left(1+\left\|\nabla u_{2 \varepsilon}\right\|_{L^{\infty}}\right)\left\|u_{\varepsilon}\right\|_{L^{2}}^{2}+\left\|\bar{g}^{\varepsilon}\right\|_{L^{2}}^{2} .
\end{aligned}
$$

Notice now that $\bar{g}^{\varepsilon} \in L^{2}\left(0, T ; L^{2}(\Omega)\right)$ and

$$
\int_{0}^{T}\left\|\bar{g}^{\varepsilon}(s)\right\|_{L^{2}}^{2} d s \leq C(J) R\|\bar{\varphi}(T)\|_{Z}^{2} .
$$

Since $u_{\varepsilon}$ is smooth owing to (3.77), we can infer from (3.88) and the application of Gronwall's inequality, that

$$
\left\|u_{\varepsilon}(t)\right\|_{L^{2}}^{2} \leq C(\varepsilon, J, R, T)\|\bar{\varphi}(t)\|_{Z}^{2}, t \in(0, T) .
$$

In fact, the constant on the right-hand side of (3.90) can be chosen independently of $\varepsilon$, but this is not much relevant at this point. On the other hand, multiplying (3.85) scalarly $B_{N}^{-1}\left(\varphi_{\varepsilon}-\left\langle\varphi_{\varepsilon}\right\rangle\right)$ (notice that we also have $\left\langle\varphi_{\varepsilon}(t)\right\rangle=\left\langle\varphi_{\varepsilon}(0)\right\rangle \equiv 0$ ). After standard transformations as in the proof of Theorem 3.8, we deduce

$$
\frac{d}{d t}\left\|\varphi_{\varepsilon}\right\|_{\left(W^{1,2}\right)^{*}}^{2}+2\left(a(x) \varphi_{\varepsilon}+F^{\prime}\left(\varphi_{1 \varepsilon}\right)-F^{\prime}\left(\varphi_{2 \varepsilon}\right), \varphi_{\varepsilon}\right) \leq 2\left|J_{1}+J_{2}+J_{3}\right|,
$$

where

$$
J_{1}=-\left(u_{2 \varepsilon} \cdot \nabla \varphi_{\varepsilon}, B_{N}^{-1} \varphi_{\varepsilon}\right), J_{2}=-\left(u_{\varepsilon} \cdot \nabla \varphi_{1 \varepsilon}, B_{N}^{-1} \varphi_{\varepsilon}\right), J_{3}=\left(J * \varphi_{\varepsilon}, B_{N}^{-1} \varphi_{\varepsilon}\right) .
$$

We can estimate the terms $J_{1}-J_{3}$ exactly as in (3.70)-(3.71), to deduce

$$
\begin{aligned}
|J 3| & \leq\left\|\nabla J * \varphi_{\varepsilon}\right\|_{L^{2}}\left\|\nabla B_{N}^{-1} \varphi_{\varepsilon}\right\| \\
& \leq \epsilon\left\|\varphi_{\varepsilon}\right\|_{L^{2}}^{2}+C(J, \epsilon)\left\|\varphi_{\varepsilon}\right\|_{\left(W^{1,2}\right)^{*}}^{2}, \\
\left|J_{1}+J_{2}\right| & \leq \epsilon\left\|\varphi_{\varepsilon}\right\|_{L^{2}}^{2}+\left\|u_{\varepsilon}\right\|_{L^{2}}^{2}+C(J, \epsilon, R)\left\|\varphi_{\varepsilon}\right\|_{\left(W^{1,2}\right)^{*}}^{2}
\end{aligned}
$$

These estimates together with (3.91) and (3.90) then yield

$$
\left\|\varphi_{\varepsilon}(t)\right\|_{\left(W^{1,2}\right)^{*}}^{2} \leq C(T, R, J, \varepsilon)\|\bar{\varphi}(t)\|_{Z}^{2}, t \in(0, T),
$$

which is the required property (3). By these considerations we can then conclude the entire thesis of this contribution.

\section{REFERENCES}

[1] H. Abels, On a diffusive interface model for two-phase flows of viscous, incompressible fluids with matched densities, Arch. Ration. Mech. Anal. 194 (2009), 463-506.

[2] H. Abels, Longtime behavior of solutions of a Navier-Stokes/Cahn-Hilliard system, Proceedings of the Conference "Nonlocal and Abstract Parabolic Equations and their Applications", Bedlewo, Banach Center Publ. 86 (2009), 9-19.

[3] J. P. Bourguignon, H. Brezis, Remarks on the Euler equation, J. Funct. Anal. 15 (1975), 341-363.

[4] P.W. Bates, J. Han, The Neumann boundary problem for a nonlocal Cahn-Hilliard equation, J. Differential Equations 212 (2005), 235-277.

[5] F. Boyer, Mathematical study of multi-phase flow under shear through order parameter formulation, Asymptot. Anal. 20 (1999), 175-212.

[6] C. Bardos, E. Titi, Mathematics and turbulence: where do we stand?, J. of Turbulence 14 (2013), doi: 10.1080/14685248.2013.771838.

[7] C. Cao, C.G. Gal, Global solutions for the 2D NS-CH model for a two-phase flow of viscous, incompressible fluids with mixed partial viscosity and mobility, Nonlinearity 25 (2012), 32113234 . 
[8] P. Colli, S. Frigeri, M. Grasselli, Global existence of weak solutions to a nonlocal CahnHilliard-Navier-Stokes system, J. Math. Anal. Appl. 386 (2012), 428-444.

[9] S. Frigeri, M. Grasselli, Global and trajectories attractors for a nonlocal Cahn-Hilliard-NavierStokes system, J. Dynam. Differential Equations 24 (2012), 827-856.

[10] S. Frigeri, M. Grasselli, Nonlocal Cahn-Hilliard-Navier-Stokes systems with singular potentials, Dyn. Partial Differ. Equ. 9 (2012), 273-304.

[11] S. Frigeri, C.G. Gal, M. Grasselli, On nonlocal Cahn-Hilliard-Navier-Stokes systems in two dimensions, to appear in J. Nonlinear Science; arXiv:1401.7954v2.

[12] S. Frigeri, M. Grasselli, P. Krejčí, Strong solutions for two-dimensional nonlocal CahnHilliard-Navier-Stokes systems, J. Differential Equations 255 (2013), 2597-2614.

[13] S. Frigeri, M. Grasselli, E. Rocca, A diffuse interface model for two-phase incompressible flows with nonlocal interactions and nonconstant mobility, Nonlinearity 28 (2015), 1257-1293.

[14] C.G. Gal, M. Grasselli, Asymptotic behavior of a Cahn-Hilliard-Navier-Stokes system in 2D, Ann. Inst. H. Poincaré Anal. Non Linéaire 27 (2010), 401-436.

[15] C.G. Gal, M. Grasselli, Trajectory attractors for binary fluid mixtures in 3D, Chinese Ann. Math. Ser. B 31 (2010), 655-678.

[16] C.G. Gal, M. Grasselli, Instability of two-phase flows: a lower bound on the dimension of the global attractor of the Cahn-Hilliard-Navier-Stokes system, Phys. D 240 (2011), 629-635.

[17] C.G. Gal, M. Grasselli, Longtime behavior of nonlocal Cahn-Hilliard equations, Discrete Contin. Dyn. Syst. Ser. A 34 (2014), 145-179.

[18] C.G. Gal, T.T. Medjo, Regularized family of models for incompressible Cahn-Hilliard twophase flows, Nonlinear Anal. Real World Appl. 23 (2015), 94-122.

[19] C.G. Gal, T.T. Medjo, On a regularized family of models for homogeneous incompressible two-phase flows, J. Nonlinear Sci. 24 (2014), no. 6, 1033-1103.

[20] H. Gajewski, K. Zacharias, On a nonlocal phase separation model, J. Math. Anal. Appl. 286 (2003), 11-31.

[21] G. Giacomin, J.L. Lebowitz, Exact macroscopic description of phase segregation in model alloys with long range interactions, Phys. Rev. Lett. 76 (1996), 1094-1097.

[22] G. Giacomin, J.L. Lebowitz, Phase segregation dynamics in particle systems with long range interactions. I. Macroscopic limits, J. Statist. Phys. 87 (1997), 37-61.

[23] C. Liu, J. Shen, A phase field model for the mixture of two incompressible fluids and its approximation by a Fourier spectral method, Phys. D 179 (2003), 211-228.

[24] S.-O. Londen, H. Petzeltová, Convergence of solutions of a non-local phase-field system, Discrete Contin. Dyn. Syst. Ser. S 4 (2011), 653-670.

[25] S.-O. Londen, H. Petzeltová, Regularity and separation from potential barriers for a non-local phase-field system, J. Math. Anal. Appl. 379 (2011), 724-735.

[26] J.S. Kim, Phase-field models for multi-component fluid flows, Commun. Comput. Phys. 12 (2012), 613-661.

[27] V.N. Starovoitov, The dynamics of a two-component fluid in the presence of capillary forces, Math. Notes 62 (1997), 244-254.

[28] L. Zhao, H. Wu, H. Huang, Convergence to equilibrium for a phase-field model for the mixture of two viscous incompressible fluids, Commun. Math. Sci. 7 (2009), 939-962.

[29] V.I. Yudovich, Uniqueness theorem for the basic nonstationary problem in the dynamics of an ideal incompressible fluid, Math. Res. Lett. 2 (1995), no. 1, 27-38.

Department of Mathematics \& Statistics, Florida International University, MiAMI, FL 33199, USA

E-mail address: cgal@fiu.edu 